\title{
The dependence of oxygen and nitrogen abundances on stellar mass from the CALIFA survey $\star, \star \star$
}

E. Pérez-Montero ${ }^{1}$, R. García-Benito ${ }^{1}$, J. M. Vílchez ${ }^{1}$, S. F. Sánchez ${ }^{1,2}$, C. Kehrig ${ }^{1}$, B. Husemann ${ }^{3}$, S. Duarte Puertas ${ }^{1}$, J. Iglesias-Páramo ${ }^{1}$, L. Galbany ${ }^{4,5}$, M. Mollá ${ }^{6}$, C. J. Walcher ${ }^{3}$, Y. Ascasíbar ${ }^{7}$, R. M. González Delgado ${ }^{1}$, R. A. Marino ${ }^{8,9}$, J. Masegosa ${ }^{1}$, E. Pérez ${ }^{1}$, F. F. Rosales-Ortega ${ }^{10}$, P. Sánchez-Blázquez ${ }^{7,11}$, J. Bland-Hawthorn ${ }^{12}$, D. Bomans ${ }^{13}$, Á. R. López-Sánchez ${ }^{14,15}$, B. Ziegler ${ }^{16}$, and The CALIFA Collaboration

1 Instituto de Astrofísica de Andalucía - CSIC, Apdo. de correos 3004, 18080 Granada, Spain e-mail: epm@iaa.es

2 Instituto de Astronomía, Universidad Nacional Autonóma de Mexico, A.P. 70-264, 04510 México, D.F., Mexico

3 Leibniz-Institut für Astrophysik Potsdam (AIP), An der Sternwarte 16, 14482 Potsdam, Germany

4 Millennium Institute of Astrophysics, Chile

5 Departamento de Astronomía, Universidad de Chile, Camino El Observatorio 1515, Las Condes, Santiago, Chile

${ }^{6}$ Departamento de Investigación Básica, CIEMAT, Avda. Complutense 40, 28040 Madrid, Spain

7 Departamento de Física Teórica, Universidad Autónoma de Madrid, 28049 Madrid, Spain

8 Department of Physics, Institute for Astronomy, ETH Zürich, 8093 Zürich, Switzerland

9 Departamento de Astrofísica y CC. de la Atmósfera, Facultad de CC. Físicas, Universidad Complutense de Madrid, Avda. Complutense s/n, 28040 Madrid, Spain

10 Instituto Nacional de Astrofísica, Óptica y Electrónica, Luis E. Erro 1, 72840 Tonantzintla, Puebla, Mexico

11 Instituto de Astrofísica, Facultad de Física, Pontificia Universidad Católica de Chile, 4860 Av. Vicuña Mackenna, Santiago, Chile

12 Sydney Institute for Astronomy, School of Physics A28, University of Sydney, NSW 2006, Australia

13 Astronomical Institute of the Ruhr-University Bochum Universitaetsstr. 150, 44801 Bochum, Germany

14 Australian Astronomical Observatory, PO Box 915, North Ryde, NSW 1670, Australia

15 Department of Physics and Astronomy, Macquarie University, NSW 2109, Australia

16 University of Vienna, Türkenschanzstrasse 17, 1180 Vienna, Austria

Received 29 March 2016 / Accepted 12 July 2016

\section{ABSTRACT}

Context. The study of the integrated properties of star-forming galaxies is central to understand their formation and evolution. Some of these properties are extensive and therefore their analysis require totally covering and spatially resolved observations. Among these properties, metallicity can be defined in spiral discs by means of integral field spectroscopy (IFS) of individual H II regions. The simultaneous analysis of the abundances of primary elements, as oxygen, and secondary, as nitrogen, also provides clues about the star formation history and the processes that shape the build-up of spiral discs.

Aims. Our main aim is to analyse simultaneously $\mathrm{O} / \mathrm{H}$ and $\mathrm{N} / \mathrm{O}$ abundance ratios in $\mathrm{H}$ II regions in different radial positions of the discs in a large sample of spiral galaxies to obtain the slopes and the characteristic abundance ratios that can be related to their integrated properties.

Methods. We analysed the optical spectra of individual selected H II regions extracted from a sample of 350 spiral galaxies of the CALIFA survey. We calculated total $\mathrm{O} / \mathrm{H}$ abundances and, for the first time, N/O ratios using the semi-empirical routine HIICHI-MISTRY, which, according to Pérez-Montero (2014, MNRAS, 441, 2663), is consistent with the direct method and reduces the uncertainty in the $\mathrm{O} / \mathrm{H}$ derivation using $[\mathrm{N}$ II] lines owing to the dispersion in the $\mathrm{O} / \mathrm{H}-\mathrm{N} / \mathrm{O}$ relation. Then we performed linear fittings to the abundances as a function of the de-projected galactocentric distances.

Results. The analysis of the radial distribution both for $\mathrm{O} / \mathrm{H}$ and $\mathrm{N} / \mathrm{O}$ in the non-interacting galaxies reveals that both average slopes are negative, but a non-negligible fraction of objects have a flat or even a positive gradient (at least $10 \%$ for $\mathrm{O} / \mathrm{H}$ and $4 \%$ for $\mathrm{N} / \mathrm{O}$ ). The slopes normalised to the effective radius appear to have a slight dependence on the total stellar mass and the morphological type, as late low-mass objects tend to have flatter slopes. No clear relation is found, however, to explain the presence of inverted gradients in this sample, and there is no dependence between the average slopes and the presence of a bar. The relation between the resulting $\mathrm{O} / \mathrm{H}$ and $\mathrm{N} / \mathrm{O}$ linear fittings at the effective radius is much tighter (correlation coefficient $\rho_{\mathrm{s}}=0.80$ ) than between $\mathrm{O} / \mathrm{H}$ and $\mathrm{N} / \mathrm{O}$ slopes $\left(\rho_{\mathrm{s}}=0.39\right)$ or for $\mathrm{O} / \mathrm{H}$ and $\mathrm{N} / \mathrm{O}$ in the individual $\mathrm{H}$ II regions $\left(\rho_{\mathrm{s}}=0.37\right)$. These $\mathrm{O} / \mathrm{H}$ and $\mathrm{N} / \mathrm{O}$ values at the effective radius also correlate very tightly (less than 0.03 dex of dispersion) with total luminosity and stellar mass. The relation with other integrated properties, such as star formation rate, colour, or morphology, can be understood only in light of the found relation with mass.

Key words. ISM: abundances - galaxies: star formation - galaxies: abundances - galaxies: evolution

\footnotetext{
* Based on observations collected at the Centro Astronómico Hispano Alemán (CAHA) at Calar Alto, operated jointly by the Max-Planck Institut für Astronomie and the Instituto de Astrofísica de Andalucía (CSIC).

$\star \star$ Full Table 1 is only available at the CDS via anonymous ftp to cdsarc.u-strasbg.fr $(130.79 .128 .5)$ or via

http://cdsarc.u-strasbg.fr/viz-bin/qcat?J/A+A/595/A62
} 


\section{Introduction}

The study of galaxy formation and evolution through different cosmological epochs is currently a hot topic of research. The amount of available data coming from large ground- and spacebased telescopes at increasingly higher redshift $(z)$ and better spatial resolution is growing substantially thanks to many deep sky surveys, such as zCOSMOS (Lilly et al. 2007), MASSIV (Contini et al. 2012), MANGA (Bundy et al. 2015), or SAMI (Bryant et al. 2015). Among the different integrated properties of galaxies to investigate, metal content is of special interest to understand the evolutionary status and pathway followed towards the current state of galaxies. Metallicity $(Z)$ is a crucial parameter since its value is the fruit of the conversion of unprocessed gas onto stars, which transform light into heavier elements and spew these elements out into the interstellar medium (ISM) or even into the intergalactic medium (IGM) to take potentially part in a new loop of star formation and chemical enrichment. Then, $Z$ traces the age, star formation history, and efficiency of galaxies, and it is therefore linked to their other integrated properties (e.g. Roberts \& Haynes 1994). A thorough dissection of the observed integrated properties in the galaxies of the local Universe gives us the opportunity to look deeper into the fundamental relations observed in a Universe at larger $z$ under a new light.

In this way, the integrated values of extensive properties and the spatial distribution of intensive properties, which are not always homogeneous, can be investigated in local galaxies. In the case of $Z$, it is known to correlate with the total luminosity (e.g. Lequeux et al. 1979; Skillman 1989; Lamareille et al. 2004) and the total stellar mass of galaxies (e.g. Tremonti et al. 2004). These correlations were found to exist even in spiral galaxies, where spectroscopy of individual HII regions helps to trace a negative gradient of $Z$ (e.g. Garnett \& Shields 1987; Vila-Costas \& Edmunds 1992; Zaritsky et al. 1994; Marino et al. 2012). Despite the slopes, these gradients are found to be steeper for fainter galaxies (Garnett et al. 1997). A common mass-independent slope can be obtained when the slope is divided by the effective or the isophotal radius (e.g. Diaz 1989; Sánchez et al. 2014) and one can recover for each galaxy a characteristic $Z$ value that also correlates with their integrated properties (e.g. Pilyugin et al. 2004; Moustakas \& Kennicutt 2006). This normalisation has been also used by several authors to propose a common slope for all non-interacting disc galaxies (e.g. Bresolin et al. 2009; Rosales-Ortega et al. 2011; Bresolin et al. 2012; Sánchez et al. 2014; Ho et al. 2015; Marino et al. 2016). This could be consistent with a common inside-out scenario of growth of the disc owing to the fall of gas from the halo in all galaxies (e.g. Pichon et al. 2011).

However there are some indications that the steepness of the gradient of $Z$ in galaxy discs, and hence the derivation of a characteristic value, can be influenced by other factors. Among these, the presence of a bar or the morphological type has been proposed (e.g. Vila-Costas \& Edmunds 1992; Zaritsky et al. 1994), although also see Sánchez et al. (2014). There is also evidence for the presence of flat or even inverted gradients in interacting galaxies (e.g. Rich et al. 2012; López-Sánchez et al. 2015) or in some low-mass and/or gas-rich galaxies (e.g. Werk et al. 2010, 2011; Moran et al. 2012). On the other hand, Carton et al. (2015) find steeper gradients in gas-rich galaxies. According to the study of the radial distribution of metals at higher $z$ the fraction of galaxies with an inverted or a flat gradient could be higher owing to a higher rate of interactions and mergers in a denser
Universe (Cresci et al. 2010; Queyrel et al. 2012; Troncoso et al. 2014).

When $Z$ is obtained from the optical spectra of $\mathrm{H}$ II regions it is usually expressed in terms of the abundance of a primary element as oxygen $(12+\log (\mathrm{O} / \mathrm{H})$, hereafter $\mathrm{O} / \mathrm{H})$. Nevertheless, exploring the spatial variation of the chemical abundance ratio of a primary element or a secondary element, such as nitrogen $(\log (\mathrm{N} / \mathrm{O})$, hereafter $\mathrm{N} / \mathrm{O})$ at same time, can shed some light on the origin of the flattening or the inversion of chemical gradients in discs and the global relations with the galactic integrated properties. Edmunds (1990) shows that this kind of ratio could be relatively unaffected by hydrodynamical effects, such as infalls of metal-poor gas or outflows of enriched material. This makes the relation between N/O and the integrated stellar mass (hereafter MNOR; Pérez-Montero \& Contini 2009) correlate even in those galaxies whose $Z$ is dissevered from its star formation history (Köppen \& Hensler 2005), such as is the case in some extreme emission line galaxies (e.g. Amorín et al. 2010; Pérez-Montero et al. 2011). In this way the MNOR, contrary to the mass-metallicity relation (MZR), does not have any dependence on the integrated star formation rate (SFR; Pérez-Montero et al. 2013).

Besides the study of the spatial variation of N/O can help to derive more accurate abundance gradients in spatially resolved galaxies. Since N spreads over a much wider range of variation than O (e.g. Thuan et al. 2010) its study can be achieved at a better precision from the analysis of the emission lines from $\mathrm{H}$ II regions. In addition, possible deviations from the typical $\mathrm{O} / \mathrm{H}-\mathrm{N} / \mathrm{O}$ relation (i.e. growing $\mathrm{N} / \mathrm{O}$ for growing $\mathrm{O} / \mathrm{H}$ in the production regime of secondary $\mathrm{N}$ ) can also be useful to explore variations in the star formation efficiency (Mollá et al. 2006), the stellar yields, or initial mass function (IMF) changes across discs (e.g. Mattsson 2009), as most $\mathrm{O}$ is produced by short-living massive stars and most of $\mathrm{N}$ is ejected to the ISM by long-living low- and intermediate-mass stars in the metal-rich discs of galaxies.

Inasmuch as we aim to investigate the behaviour of the abundance gradients in local galaxies, we need a sufficiently large sample of objects covered in two dimensions with integral field spectroscopy (IFS) in the optical range, as is the case of CALIFA (Calar Alto Legacy Integral Field Area survey; Sánchez et al. 2012a). The sample of galaxies studied in the CALIFA survey is well characterised and all integrated properties are well studied and can be correlated with the resulting characteristic chemical abundances. In Sect. 2 of this paper we describe the CALIFA survey and how individual $\mathrm{H}$ II regions were extracted from the data cubes.

The rest of the paper is organised as follows: in Sect. 3.1 we give the details of the stellar continuum subtraction, emissionline measurement and, in Sect. 3.2, the selection of star-forming $\mathrm{H}$ II regions. The determination of $\mathrm{O} / \mathrm{H}$ and $\mathrm{N} / \mathrm{O}$ abundances is described in Sect. 3.3. This task was carried out using the model-based, semi-empirical code HII-CHI- MISTRY. As shown in Pérez-Montero (2014), this method is totally consistent with the direct method when it is compared with H II regions with available empirical electron temperatures. This method allows the simultaneous study of $\mathrm{O} / \mathrm{H}$ and $\mathrm{N} / \mathrm{O}$ abundance ratios without assuming that any previous relation between them is taken from a calibration sample, as occurs when a strong-line method is used based on [N II] emission lines. The global relation between $\mathrm{O} / \mathrm{H}$ and $\mathrm{N} / \mathrm{O}$ for all $\mathrm{H}$ II regions is presented in Sect. 4. In Sect. 5 we describe the calculations of radial chemical variations fittings and the statistical distributions of the resulting slopes and the values at the effective radius. We also analyse the possible correlations between the resulting slopes, the characteristic 
abundances, and other integrated properties of the galaxies, such as luminosity, stellar mass, SFR, colour, morphological type, and the presence of a bar in Sect. 6. In Sect. 7, we also discuss in detail the case of those objects with a flat or inverted $Z$ gradient. Finally, in Sect. 8, we summarise our results and present our conclusions.

\section{Sample of galaxies and extraction of $\mathrm{HII}$ regions}

The galaxies observed by CALIFA were selected from the SDSS survey encompassing a wide range of their integrated properties, including mass, luminosity, colours, and morphologies (Walcher et al. 2014). This gives us insight into many of their spatially resolved properties with a statistically significant point of view in the local Universe $(0.005<z<0.03)$.

The details of the survey, sample, observational strategy, and reduction are explained in Sánchez et al. (2012a) and García-Benito et al. (2015). All galaxies were observed using PMAS (Roth et al. 2005) in the PPAK configuration (Kelz et al. 2006), covering a hexagonal field of view (FoV) of $74^{\prime \prime} \times$ 64 " sufficient to map the full optical extent of the galaxies up to two to three disc effective radii. This is possible because of the diameter selection of the sample (Walcher et al. 2014). The observing strategy guarantees a complete coverage of the FoV with a final pixel size $1^{\prime \prime}$, corresponding to $\approx 1 \mathrm{kpc}$ at the average $z t$ of the survey.

The CALIFA mother sample consists of 939 galaxies, but for this work we used a sample of 350 objects observed with the gratings V500 and V1200. The observed wavelength range and spectroscopic resolution (3745-7500 $\AA, \lambda / \Delta \lambda \approx 850$, for the low-resolution setup and 3650-4800 $\AA, \lambda / \Delta \lambda \approx 1500$, for the high resolution setup) are more than sufficient to explore the most prominent ionised gas emission lines, from [O II] $] 3727$ to [S II] $\lambda 6731$, on the one hand, and to deblend and subtract the underlying stellar population, on the other (e.g. Sánchez et al. 2012a; Kehrig et al. 2012; Cid Fernandes et al. 2013). The dataset was reduced using version 1.5 of the CALIFA pipeline, whose modifications with respect to the pipeline presented in Sánchez et al. (2012a) are described in detail in García-Benito et al. (2015). In summary, the data fulfill the predicted quality-control requirements with a spectrophotometric accuracy that is better than $15 \%$ everywhere within the wavelength range, both absolute and relative with a depth that allows us to detect emission lines in individual $\mathrm{H}$ II regions as weak as $\approx 10^{-17} \mathrm{erg} \mathrm{s}^{-1} \mathrm{~cm}^{-2}$, and with a signal-to-noise ratio of $S / N \approx 3-5$, even in the case of [O III] auroral lines presented in Marino et al. (2013).

The final product of the data reduction is a regular-grid data cube with $x$ and $y$ coordinates that indicate the right ascension and declination of the target and $z$ a common step in wavelength. The CALIFA pipeline also provides the propagated error cube, a proper mask cube of bad pixels, and a prescription of how to handle the errors when performing spatial binning (due to covariance between adjacent pixels after image reconstruction). These data cubes, together with the ancillary data described in Walcher et al. (2014), are the basic starting points of our analysis.

Emission-line regions in the CALIFA galaxies were segregated in the resulting data cubes and their corresponding spectra were extracted using the semi-automatic routine HIIEXPLORER ${ }^{1}$. The details of this routine are described well in Sánchez et al. (2012b) and Rosales-Ortega et al. (2012), but

\footnotetext{
Available at http://www . caha.es/sanchez/HII_explorer
}

basically the routine is based on the assumption that emissionline regions can be found as clumpy and isolated $\mathrm{H} \alpha$ peaks of emission of several arcseconds, which correspond to diameters of a few hundreds of parsecs. The total number of selected emission-line regions from the 350 analysed galaxies following this procedure is 15757 .

\section{Analysis of the extracted regions}

\subsection{Emission-line measurement}

The stellar underlying population spectral emission was fitted and removed from the extracted individual spectra of the extracted emission-line regions to avoid absorption components of the Balmer emission lines. This continuum was fitted using the programme FIT3D v. $2.0^{2}$ (Sánchez et al. 2006, 2011). This package fits the continuum as a linear combination of several single stellar populations (SSPs) of different ages and $Z$ taken from the MILES project (Vazdekis et al. 2010; Falcón-Barroso et al. 2011). A stellar extinction law by Cardelli et al. (1989) was used in the fitting process, assuming a value of $R_{v}=3.1$, a simple dust screen distribution, and that all SSPs have the same dust attenuation.

Once the stellar continuum is subtracted, FIT3D performed a Gaussian function fitting to measure the fluxes of the most prominent emission lines of the extracted spectra. To reduce the number of free parameters, this algorithm forces the systemic velocity and the velocity dispersion to be the same for emission lines produced by the same ion (e.g. [O III] 4959, $5007 \AA$ ) and to apply a multi-component analysis for those blended emission lines. For the aim of this work, we used [O II] $3727 \AA$, $\mathrm{H} \beta$, [O III] $5007 \AA, \mathrm{H} \alpha$, [N II] $6584 \AA$, and [S II] 6717, $6731 \AA$ with a signal-to-noise ratio better than 3 as obtained using the above described automatic fitting procedure.

\subsection{Selection of star-forming $H$ II regions}

The determination of chemical abundances and most of the physical properties in ionised gaseous regions are based on the assumption that the gas is ionised by a spectral energy distribution dominated by very young massive stars during a process of ongoing star formation. Therefore, for the extracted objects in our sample of CALIFA galaxies, it is necessary to separate starforming regions from other emission-line regions in which the main source of ionisation is different (i.e. AGNs, shocks, and post-AGB stars).

The most widely used method based on optical emission lines to detect star-forming regions is based on diagnostic diagrams of emission-line ratios sensitive to the excitation of the gas (e.g. Baldwin et al. 1981 (BPT); Veilleux \& Osterbrock 1987). One of these diagrams is the extinction-independent relation between [O III] $5007 \AA$ $/ \mathrm{H} \beta$ and [N II] $6584 \AA$ $/ \mathrm{H} \alpha$, where different demarcation curves are used to classify the ionising source. Figure 1 shows this diagram for the 15757 extracted emissionline regions in the 350 analysed CALIFA galaxies and the empirical curve defined by Kauffmann et al. (2003) using SDSS galaxies and the model-based curve defined by Kewley et al. (2001). There is a general consensus that below these two lines, emission-line objects can be considered star-forming regions, while those above both curves are ionised by an AGN or shocks.

2 Available at http://www.astroscu.unam.mx/ sfsanchez/ FIT3D/index.html 


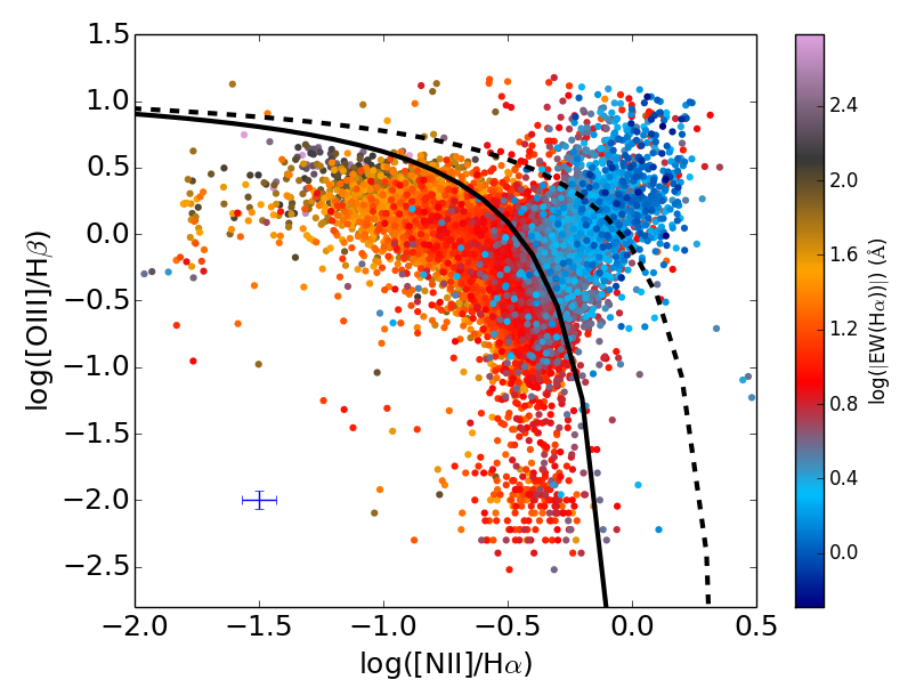

Fig. 1. $[\mathrm{O} \mathrm{III}] / \mathrm{H} \beta$ against $[\mathrm{N} \mathrm{II}] / \mathrm{H} \alpha$ relation, one of the BPT diagrams, for the 15757 extracted emission-line regions in the analysed CALIFA galaxies. Colour scale shows the $\mathrm{H} \alpha$ equivalent width. The solid black line represents the empirical curve from Kauffmann et al. (2003) to divide objects ionised by massive stars and by non-thermal processes. The dashed line is the theoretical curve defined by Kewley et al. (2001). The lower left cross indicates the typical associated error in each axes.

All regions in that part of the diagram were discarded for the subsequent analysis.

In addition, the regions between these two curves were proposed to be composite regions in which their ionisation has a mixed origin (i.e. star formation and nuclear activity) (Kewley et al. 2006), although we can also find pure starforming regions with an overabundance of nitrogen, as in Pérez-Montero \& Contini (2009), or regions that can be ionised by evolved stars (Kehrig et al. 2012). Therefore, to select only star-forming $\mathrm{H}$ II regions we also used the criterion described by Cid Fernandes et al. (2011) based on the use of $\mathrm{EW}(\mathrm{H} \alpha)$, as a proxy of the weight of the old stellar population. This was measured on the spectra before the subtraction of the underlying stellar population over the continuum. As shown in Fig. 1 the distribution of $E W(\mathrm{H} \alpha)$ correlates with the position of the regions in the BPT diagram. In this way, as also carried out by Sánchez et al. (2014), we discarded for our analysis those regions with $E W(\mathrm{H} \alpha)>-6 \AA$. In addition, to ensure that only regions ionised by massive star formation are used, we took the results from the SSP fitting to select regions with at least a mass fraction of $30 \%$ of young stars (i.e. with an age younger than $500 \mathrm{Myr}$ ). The number of selected star-forming regions in the 350 analysed CALIFA galaxies using the above criteria is then 8196 .

\subsection{Derivation of chemical abundances}

Both elemental abundance ratios $\mathrm{O} / \mathrm{H}$ and $\mathrm{N} / \mathrm{O}$ were derived in the selected sample of $\mathrm{H}$ II regions using the programme HII-CHI-MISTRY v.2.0 $0^{3}$ (hereafter HCM) (Pérez-Montero 2014). This method calculates these abundance ratios and the ionisation parameter $(\log U)$ as the averages and standard deviations of the $1 / \chi^{2}$-weighted distributions of the input conditions in a large grid of photoionisation models calculated with CLOUDY v. 13.01 (Ferland et al. 2013). The grid of models cover a wide range in the space of $\mathrm{O} / \mathrm{H}([6.9,9.1]$ in steps of $0.1 \mathrm{dex}), \mathrm{N} / \mathrm{O}([-2.0, .0]$ in

Available at http://www.iaa.es/ epm/HII-CHI-mistry.html steps of $0.125 \mathrm{dex})$, and $\log U([-4.0,-1.5]$ in steps of $0.25 \mathrm{dex})$. To avoid the clustering of the resulting quantities around the discrete values of the grid, however, we calculated linear interpolations both in the $\mathrm{O} / \mathrm{H}$ and the N/O axis and, thereby, improved the resolution up to 0.02 dex and 0.025 dex, respectively.

The $1 / \chi^{2}$ weights are calculated as the quadratic differences between certain observed emission-line ratios and the predictions made for the same ratios in each model of the grid. The observational input consists of the reddening-corrected relative-to$\mathrm{H} \beta$ fluxes of [O II] $3727 \AA$, [O III] 4363, $5007 \AA$, [N II] $6584 \AA$, and [S II] $6717+6731 \AA$. The extinction correction of all lines was applied comparing the observed $\mathrm{H} \alpha / \mathrm{H} \beta$ flux ratio with the theoretical value predicted by Storey \& Hummer (1995) for standard conditions of density and temperature (i.e. $\mathrm{H} \alpha / \mathrm{H} \beta=$ 2.86 for $T_{\mathrm{e}}=10^{4} \mathrm{~K}$ and $n_{\mathrm{e}}=10^{2} \mathrm{~cm}^{-3}$ ) and assuming an extinction law by Cardelli et al. (1989) with $R_{v}=3.1$. We checked that the temperature and density variation across the analysed $\mathrm{H}$ II regions introduces an additional uncertainty in the extinction correction that is much lower than the uncertainties associated with the emission-line measurements.

The analysis performed by Pérez-Montero (2014) shows that when the chemical abundances derived using HCM are compared with the abundances derived using the direct method in a sample of $\mathrm{HII}$ regions and galaxies with a measurement of their electron temperatures, the agreement is much better than the associated errors. This agreement is also found even though the models use only stellar energy distributions of massive clusters from POPSTAR Mollá et al. (2009) with a single age (1 Myr). However, the consistency between the abundances predicted by the grid of models and the abundances calculated using the direct method can only be confirmed in the sample of objects with electron temperature, so for our sample this can only be fully proven when the corresponding auroral lines are measured with enough confidence.

Using $\mathrm{HCM}$ to derive both $\mathrm{O} / \mathrm{H}$ and $\mathrm{N} / \mathrm{O}$ values in $\mathrm{HII}$ regions has three important advantages over other strongline methods, whether they have been empirically or theoretically calibrated. Firstly, it derives N/O as a first step using appropriate emission-line ratios (i.e. N2O2, N2S2; Pérez-Montero \& Contini 2009), allowing the use of [N II] emission lines to calculate $\mathrm{O} / \mathrm{H}$ without any prior assumption on the $\mathrm{O} / \mathrm{H}-\mathrm{N} / \mathrm{O}$ relation. Secondly, the use of models allows us to obtain consistent chemical abundances in a $Z$ regime that cannot be properly calibrated using the direct method (i.e. oversolar abundances), which makes this method especially useful for the chemical study of H II regions in spiral discs. Finally, HCM leads to values of the abundances that are consistent with the direct method regardless of the set of emission lines taken as observed input, instead of using different strong-line methods that are possibly calibrated in different ways for different available emission lines.

Unfortunately, the auroral [O III] $4363 \AA$, which is needed to calculate electron temperatures, was not measured with enough signal-to-noise in barely any of the selected H II regions of our CALIFA sample. This line could only be measured with confidence for 16 H II regions (Marino et al. 2013). For these regions, the agreement between the abundances derived from the direct method and the abundances derived from $\mathrm{HCM}$ is better than the derived errors. For the rest of the sample, in absence of the [O III] auroral line, HCM uses a limited grid of empirically constrained models to provide chemical abundances that are consistent with the direct method for the sample of H II regions with electron temperatures described in Pérez-Montero (2014). In this 


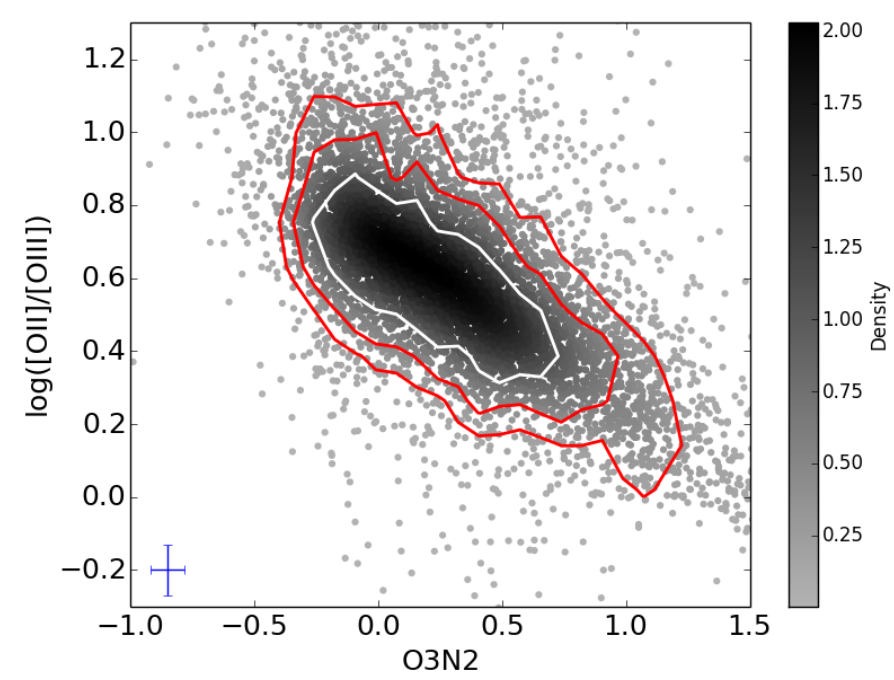

Fig. 2. Relation between the index O3N2, which is a tracer of $Z$, and the emission line ratio $[\mathrm{OII}] /[\mathrm{O} \mathrm{III}]$, which is a tracer of the excitation, for those selected star-forming regions for which [O II] lines were used for the calculation of chemical abundances. Density of points over the mean value are represented according to the colour bar. White solid line represents the $1 \sigma$ contour, while red lines represent $2 \sigma$ and $3 \sigma$ contours. The left lower cross indicates the typical errors of the two ratios.

way, it is assumed to be an empirical law between $\mathrm{O} / \mathrm{H}$ and $\log U$ (i.e. higher $\log U$ values for lower $\mathrm{O} / \mathrm{H}$ and vice versa). This $\mathrm{O} / \mathrm{H}-\log U$ relation has been already observed in different samples of H II regions (e.g. Evans \& Dopita 1985; Dopita \& Evans 1986) and could be the effect of the use of a biased sample or an evolutionary sequence for the empirical calibration of most strong-line methods. This assumption is well justified in the case of our sample as can be seen in Fig. 2, where it is represented the emission-line ratio $[\mathrm{O} \mathrm{II}] /[\mathrm{O} \mathrm{III}]$, which can be used as an indicator of the excitation of the ionised gas (e.g. Pérez-Montero \& Díaz 2005), with the O3N2 parameter $(\mathrm{O} 3 \mathrm{~N} 2=\log ([\mathrm{O} \mathrm{III}] / \mathrm{H} \beta \cdot \mathrm{H} \alpha /[\mathrm{N}$ II $]))$, which in metal-rich objects, correlates with $Z$ (e.g. Alloin et al. 1979). There is a clear trend towards lower values of [O II $] /[\mathrm{O}$ III] (high $\log U$ ) for high O3N2 (i.e. low $\mathrm{O} / \mathrm{H}$ ) and vice versa. Despite the assumed $Z-\log U$ in the models, HCM allows a certain variation of $\log U$ for each value of $\mathrm{O} / \mathrm{H}$, according to the observed relation for $\mathrm{H}$ II regions with electron temperatures in Pérez-Montero (2014) and in agreement to some expected variation of $\log U$ across galactic discs (see Ho et al. 2015) or the hardening of the ionising radiation (Pérez-Montero \& Vílchez 2009).

Using this constrained grid of models, the uncertainty in the final results is however higher than with the presence of the [O III] auroral line. We considered the following quadratic addition as the associated error in each $\mathrm{H}$ II region:

$\sigma_{\mathrm{HII}}^{2}=\sigma_{\mathrm{HCm}}^{2}+\sigma_{\mathrm{g}}^{2}+\sigma_{\mathrm{res}}^{2}$,

where $\sigma_{\mathrm{HCm}}$ is the standard deviation of the resulting distribution of abundances derived by $\mathrm{HCM}, \sigma_{\mathrm{g}}$ is the improved interpolated resolution of the grid of models (i.e. 0.02 dex for $\mathrm{O} / \mathrm{H}$ and 0.025 dex for $\mathrm{N} / \mathrm{O}$ ), and $\sigma_{\text {res }}$ is the average residual with the sample of objects with abundances derived using the direct method reported by Pérez-Montero (2014) using a sample of H II regions with measured electron temperatures (i.e. 0.22 dex for $\mathrm{O} / \mathrm{H}$ and 0.16 dex for $\mathrm{N} / \mathrm{O}$ ) in absence of [O III] $4363 \AA$ in the whole range.

Although all optical strong lines required by НCM were measured in all objects, the [O II] lines were not used for the

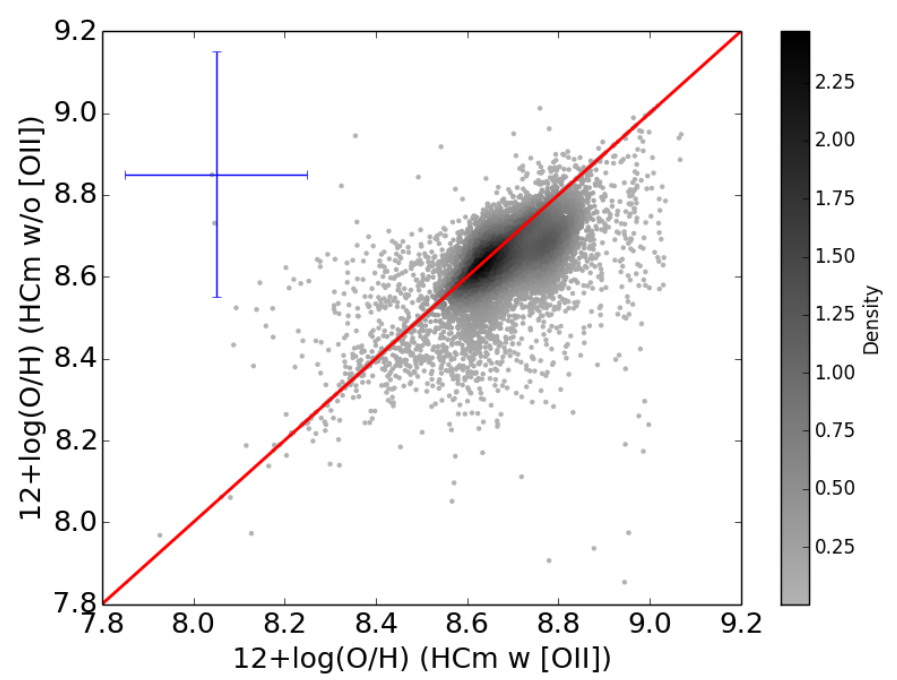

Fig. 3. Comparison between the $\mathrm{O} / \mathrm{H}$ abundances derived from $\mathrm{HCM}$ with and without taking [O II] emission lines. We only represent the $\mathrm{H}$ II regions of the sample whose $\mathrm{O} / \mathrm{H}$ uncertainty is reduced when [O II] is considered. Density of points over the mean value are represented according to the colour bar. The red solid line represents the 1:1 relation. The upper left cross represents the standard deviation of both methods in relation to the direct method as carried out by Pérez-Montero (2014).

calculation of the abundances in all of them (in a $29 \%$ of the objects). When the standard deviation of the $1 / \chi^{2}$-weighted modelbased abundances were lower without [O II], these were ruled out to reduce the resulting uncertainties (i.e. the [O II] fluxes can be more uncertain because of a more critical flux calibration in the blue part of the spectrum and to a larger wavelength baseline for reddening corrections). A comparison between the $\mathrm{O} / \mathrm{H}$ abundances derived by our method with and without [O II] for those $\mathrm{H}$ II regions whose [O II] were used is shown in Fig. 3. The mean residual is 0.04 dex, which is much lower than the reported errors, so this does not affect the results of this work very much.

A direct comparison between the chemical abundances obtained from HCM and the direct method cannot be established as the electron temperature cannot be measured in almost any $\mathrm{H}$ II regions of the CALIFA sample. However, we compare our results with some other strong-line methods calibrated with the direct method sample. The left panel of Fig. 4 shows that the comparisons between the N/O obtained from HCM with the N/O from the calibration by Pérez-Montero \& Contini (2009) of the $\mathrm{N} 2 \mathrm{~S} 2$ parameter $(=\log ([\mathrm{N} \mathrm{II}] /[\mathrm{S} \mathrm{II}]))$ for all the selected $\mathrm{H}$ II regions. The right panel shows the comparison with the N/O from the calibration by Pilyugin \& Grebel (2016) based on [N II] and [O II] lines only for those objects whose [O II] lines were used in the HCM calculations. In both cases the average of the residuals is much lower than the reported associated uncertainty (i.e. 0.05 dex in both cases), but the standard deviation of these residual results is much lower for $\mathrm{N} 2 \mathrm{~S} 2(0.10 \mathrm{dex})$ than for the method based on $[\mathrm{N} \mathrm{II}] /[\mathrm{O}$ II] calibrated by Pilyugin \& Grebel (2016) (0.25 dex).

Regarding $\mathrm{O} / \mathrm{H}$, in order to examine the impact of considering a free $\mathrm{O} / \mathrm{H}-\mathrm{N} / \mathrm{O}$ relation in the models as compared with other methods empirically calibrated, in Fig. 5 we show the comparison for the selected CALIFA star-forming regions between the $\mathrm{O} / \mathrm{H}$ derived by $\mathrm{HCM}$ and the empirical calibrations of the O3N2 parameter given by Pérez-Montero \& Contini (2009) that is valid for $\mathrm{O} / \mathrm{H} \gtrsim 8.1$. There is not a large systematic offset between the two methods (i.e. the average of the residuals is 0.04 dex) and the dispersion, which is calculated as the standard 

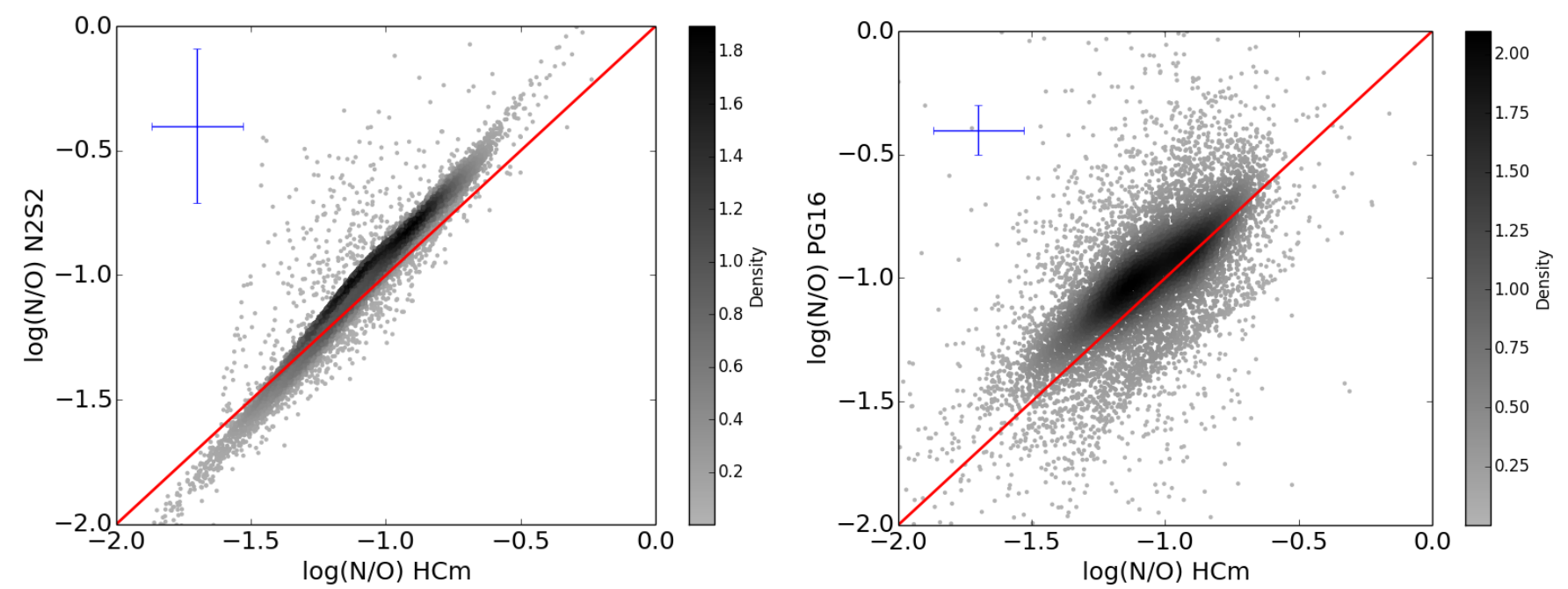

Fig. 4. Comparison between N/O derived from $\mathrm{HCM}$ and from different strong-line methods. At left, for all selected H II regions, with the N2S2 parameter calibrated by Pérez-Montero \& Contini (2009). At right, the calibration by Pilyugin \& Grebel (2016) based on [N II] and [O II] emission lines for those regions whose [O II] lines were used. Density of points over the mean value are represented according to the colour bar. The red solid line represents the 1:1 relation. The upper left crosses represent the standard deviation of the residuals to the direct method as carried out by Pérez-Montero \& Contini (2009) and Pérez-Montero (2014).
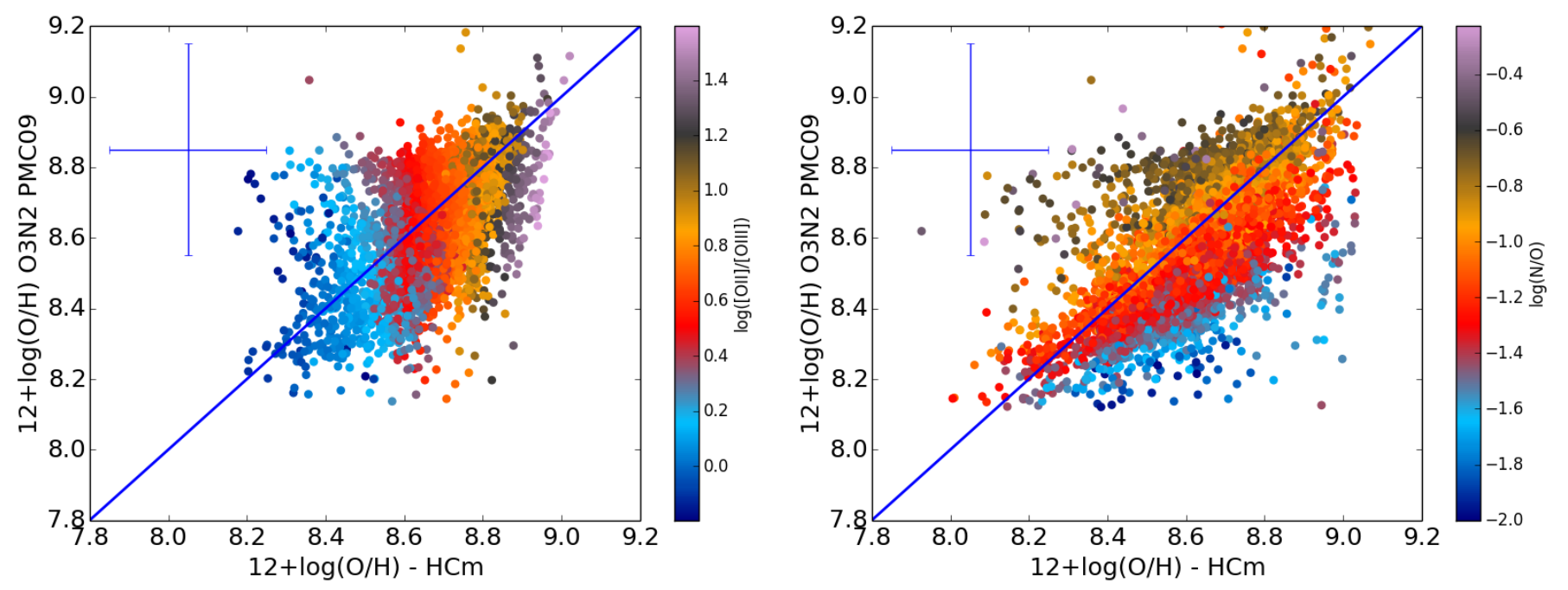

Fig. 5. Comparison between $\mathrm{O} / \mathrm{H}$ derived from $\mathrm{HCM}$ and from the empirical calibration of the $\mathrm{O} 3 \mathrm{~N} 2$ parameter given by Pérez-Montero \& Contini (2009). The blue solid lines represent the $1: 1$ relation. The left panel has a colour scale for the [O II]/[O III] ratio and the right panel for N/O. For the sake of consistency, the number of points in left panel is lower because [O II] lines were not used for the derivation of $\mathrm{O} / \mathrm{H}$. The upper left crosses represent the standard deviations of the residuals to the direct method of both methods as compared to the direct method carried out by Pérez-Montero \& Contini (2009) and Pérez-Montero (2014).

deviation of the residuals, is 0.11 dex. To explore the origin of this dispersion, we include a colour scale representing other quantities in both panels of the figure. In the left panel, only for those $\mathrm{H}$ II regions whose [O II] lines were used for the abundance calculation, the emission line ratio [O II $] /[\mathrm{O}$ III $]$, which is a tracer of the gas excitation. As in the case of Fig. 2, there is a clear trend towards higher excitation for lower $Z$. However, there is not any clear indication that the dispersion can be due to the excitation. On the other hand, in the right panel, the colour scale indicates the $\mathrm{N} / \mathrm{O}$ ratio. As can be seen the agreement is better for lower $Z$ when $\mathrm{N} / \mathrm{O}$ is lower, and for higher $\mathrm{O} / \mathrm{H}$ when $\mathrm{N} / \mathrm{O}$ is higher. Other combinations of $\mathrm{O} / \mathrm{H}$ and $\mathrm{N} / \mathrm{O}$ in the same plane leads to larger deviations of the 1:1 relation producing the observed dispersion.
In Fig. 6 we show a similar comparison with O3N2 as calibrated by Pérez-Montero \& Contini (2009) but, in this case, the $\mathrm{O} / \mathrm{H}$ abundances derived using $\mathrm{HCM}$ are derived used a grid of models assuming the expected relation between $\mathrm{O} / \mathrm{H}$ and $\mathrm{N} / \mathrm{O}$ (i.e. in the regime of secondary production of $\mathrm{N}, \mathrm{N} / \mathrm{O}$ grows with $\mathrm{O} / \mathrm{H}$; see right panel of Fig. 3 in Pérez-Montero 2014). In this case, the dispersion is very small; i.e. the standard deviation of the residuals is reduced to 0.05 dex. Since most of the objects with electron temperature used to calibrate strong-line methods follow the above typical O/H-N/O relation, the agreement is good when it is assumed. However, as in the case of our sample of CALIFA star-forming regions, the dispersion is enhanced as a consequence of a uncertain $\mathrm{N} / \mathrm{O}$ value, if it is not previously determined. 


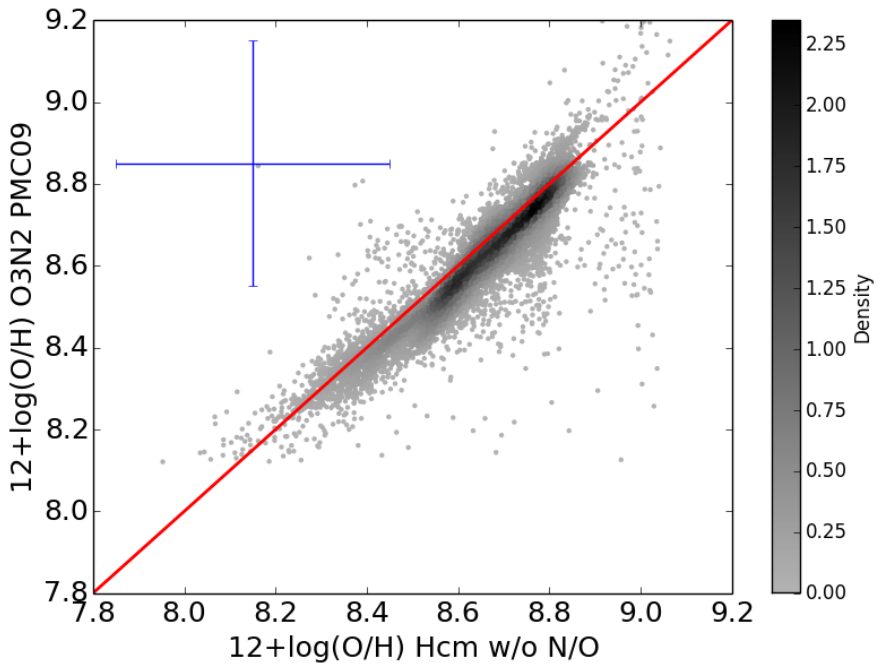

Fig. 6. Comparison between the $\mathrm{O} / \mathrm{H}$ abundances derived from $\mathrm{HCM}$ with the empirical calibration of the $\mathrm{O} 3 \mathrm{~N} 2$ parameter given by Pérez-Montero \& Contini (2009). In this case $\mathrm{HCM} \mathrm{O} / \mathrm{H}$ values were calculated without a previous determination of $\mathrm{N} / \mathrm{O}$, but assuming the expected $\mathrm{O} / \mathrm{H}-\mathrm{N} / \mathrm{O}$ relation. Density of points over the mean value are represented according to the colour bar. The red solid line represents the 1:1 relation. The upper left cross represents the standard deviations of the residuals of both methods as compared to the direct method carried out by Pérez-Montero \& Contini (2009) and Pérez-Montero (2014).

\section{4. $\mathrm{O} / \mathrm{H}-\mathrm{N} / \mathrm{O}$ for individual $\mathrm{H}$ II regions}

The relation between $\mathrm{O} / \mathrm{H}$ and N/O gives clues about the chemical evolution of the sites where the ionised gas is observed. As $\mathrm{O}$ is a primary element it is mainly ejected to the ISM by massive stars. On the contrary, $\mathrm{N}$ can have either a primary or a secondary origin. This last regime dominates the production of $\mathrm{N}$ in metal-rich $\mathrm{H}$ II regions, as it is produced via the $\mathrm{CNO}$ cycle in less massive stars. It is thus expected that N/O correlates with $\mathrm{O} / \mathrm{H}$ for the $\mathrm{H}$ II regions of the spiral discs.

Figure 7 shows the relation between $\mathrm{O} / \mathrm{H}$ and N/O calculated using HCM as described above for the 8196 star-forming regions selected among the 350 analysed CALIFA galaxies. As can be seen there is a trend towards a growing N/O for growing $\mathrm{O} / \mathrm{H}$ as corresponds to a regime of secondary production of $\mathrm{N}$ in metalrich objects.

We can compare the average position in the $\mathrm{O} / \mathrm{H}-\mathrm{N} / \mathrm{O}$ plot of the CALIFA star-forming regions analysed in this work with the line enclosing the regions and galaxies analysed in Pérez-Montero (2014) with electron temperatures, and whose $\mathrm{O} / \mathrm{H}$ and N/O abundances are also derived by Pérez-Montero (2014) to constrain the grid of models used when a previous N/O determination is not possible. We see that the slope is higher for the CALIFA regions and that the sample presents lower values of $\mathrm{N} / \mathrm{O}$ for lower $\mathrm{O} / \mathrm{H}$ values. As seen in right panel of Fig. 5, we can find some $\mathrm{H}$ II regions with oversolar $\mathrm{O} / \mathrm{H}$ and $\mathrm{N} / \mathrm{O}<-1.6$, but these can be considered as spurious given their low number $(<0.5 \%)$ and they do not affect the results regarding slopes and characteristic values described in the sections below.

We also added to Fig. 7 some $\mathrm{H}$ II regions with a measurement of their abundances following the direct method in other $Z$ ranges to check the consistency of the abundances derived in our sample. The data at high $Z$ from NGC 5194 (Croxall et al. 2015) mainly overlap our sample while low- $Z$ data from M101 (Croxall et al. 2016, and references therein) show a certain overlap with our sample, but they are in agreement with the region of

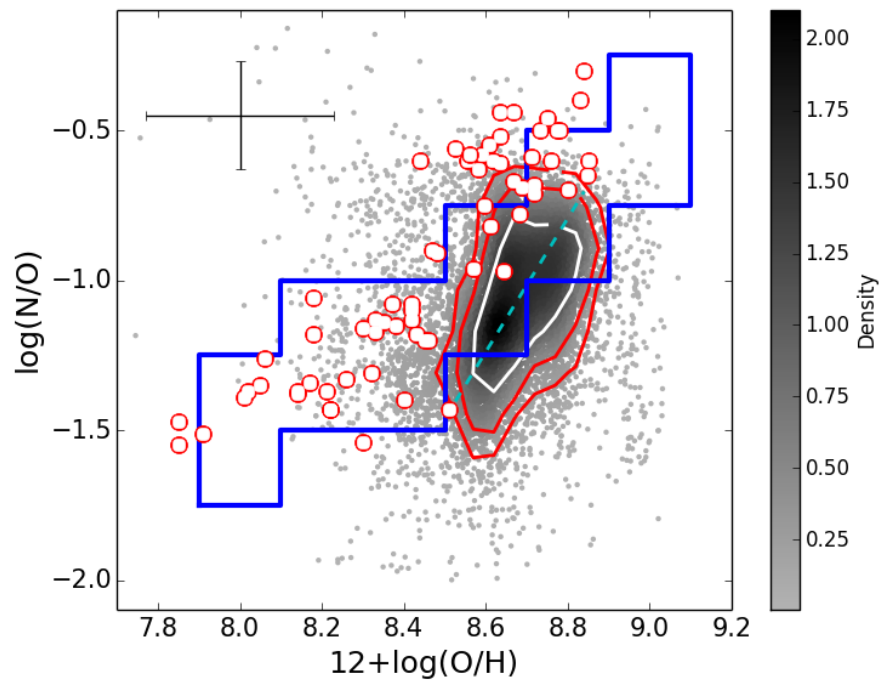

Fig. 7. Relation between $\mathrm{O} / \mathrm{H}$ and $\mathrm{N} / \mathrm{O}$ as derived from $\mathrm{HCM}$ for all the star-forming H II regions analysed in the CALIFA sample. Density of points over the mean value are coded by the colours. The solid lines represent the $1 \sigma$ (white), $2 \sigma$, and $3 \sigma$ (red) contours, while the dashed line shows the linear fitting obtained in the lower panel of Fig. 9. Finally, the cyan solid line encompasses the space of models used by HCM to calculate $\mathrm{O} / \mathrm{H}$ without a previous determination of $\mathrm{N} / \mathrm{O}$ that is taken from the position of objects with a direct chemical abundance in Pérez-Montero (2014). The upper cross represents the typical errors associated with the derivation of abundances using HCM. The white points correspond to $\mathrm{H}$ II regions in other $\mathrm{O} / \mathrm{H}$ regimes whose abundances were calculated from the direct method taken from Croxall et al. (2015, 2016).

the plot corresponding to other $\mathrm{H}$ II regions with available electron temperatures. In Fig. 8 we show, for these high- and low$Z$ H II regions, the comparison between the abundances derived from the direct method and the abundances derived from $\mathrm{HCM}$ without using [O III] $4363 \AA$, which is consistent with the derivation of the abundances in the CALIFA sample. As can be seen, no systematic offset is found either for $\mathrm{O} / \mathrm{H}$ or for $\mathrm{N} / \mathrm{O}$ with standard deviation of the residuals that is much lower than the associated uncertainties.

At the same time it can be seen that the relation between $\mathrm{O} / \mathrm{H}$ and N/O shows a large dispersion. A given dispersion is also found with theoretical models as due to the different efficiencies of SFR in the different regions or galaxies as Mollá et al. (2006) found. In that work the dispersion for the last time step calculated for their models is shown in their Fig. 8 around the fitting given for those results. However, the dispersion found with our data seems larger than that expected as due to different star formation efficiencies It is, however, necessary to take into account that the time evolution also plays a role. As it is seen in Fig. 9 from Mollá et al. (2006), different regions or galaxies evolve differently in the plane $\mathrm{O} / \mathrm{H}-\mathrm{N} / \mathrm{O}$, showing steeper tracks when the region/galaxy has higher efficiencies than those with low values, which show flat evolutionary tracks. Therefore, the data found below the region of the plane shown by the 1 sigma contours probably have medium to high efficiencies to form stars, but are less evolved than those in the "standard" locus; while the data above are probably regions with low efficiencies.

Besides, the gas interchange between a galaxy and the surrounding IGM, or between different parts of a galaxy, has a very different influence on $\mathrm{O} / \mathrm{H}$ and the ratio between the abundances of a primary and secondary element (Edmunds 1990), so the 

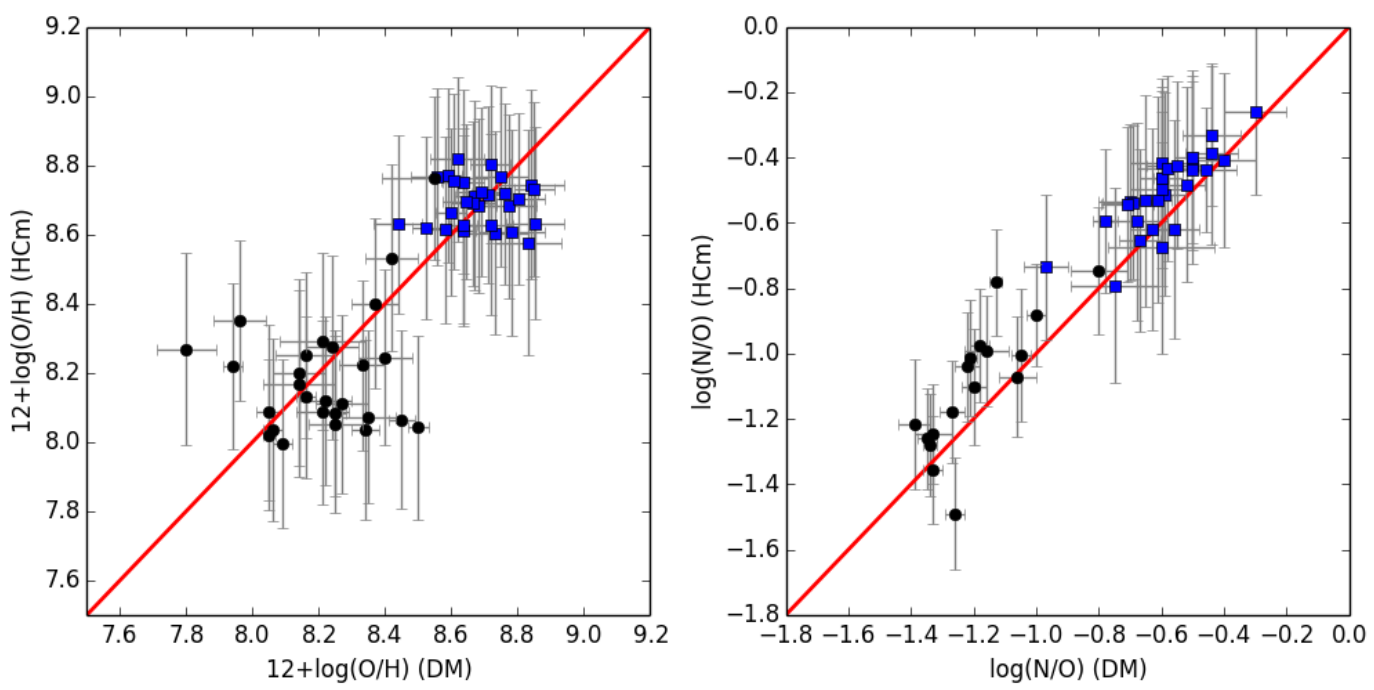

Fig. 8. Comparison between abundances derived from the direct method and from HCM in the sample of H II regions of M51 (Croxall et al. 2015) as blue squares and M101 (Croxall et al. 2016) as black circles for O/H (left) and N/O (right). The red solid lines represent the 1:1 relation in both panels.

dispersion in the $\mathrm{O} / \mathrm{H}-\mathrm{N} / \mathrm{O}$ diagram tends to be larger when gas flows exist in the galaxies.

On the other hand, this plot illustrates the importance of the determination of $\mathrm{N} / \mathrm{O}$ for the chemical analysis in spiral galaxies. Firstly, as N/O does not follow the expected trend in its relation with $\mathrm{O} / \mathrm{H}$ and presents a large dispersion, all $\mathrm{O} / \mathrm{H}$ determinations based on [N II] lines can lead to wrong results (Pérez-Montero \& Contini 2009) and, secondly, the range of variation in this sample is almost three times larger in logarithmic scale for $\mathrm{N} / \mathrm{O}(\approx[-1.8,-0.5])$ than for $\mathrm{O} / \mathrm{H}(\approx[8.4-8.9])$. In addition, in absence of the [O III] auroral line, the relative error using $\mathrm{HCM}$ is better for N/O $(0.16 \mathrm{dex})$ than for $\mathrm{O} / \mathrm{H}(0.22 \mathrm{dex})$. Thus, the variations of $\mathrm{N} / \mathrm{O}$ across galactic discs, can yield more accurate results for the determination of spatial chemical variations across galactic discs.

\section{Chemical abundance gradients in discs}

\subsection{Calculation of linear fittings}

By considering the resulting $\mathrm{HCM} \mathrm{O} / \mathrm{H}$ and $\mathrm{N} / \mathrm{O}$ abundance ratios and the de-projected radial distances of the corresponding selected star-forming regions, in our sample of 350 CALIFA galaxies, robust error-weighted linear fittings were performed.

The analysis was made at all de-projected galactocentric distances normalised to the effective radius. The description about the measurement of the effective radius in each object is given in Sánchez et al. (2014). Only those galaxies with ten or more selected $\mathrm{H}$ II regions were considered for the subsequent statistical analysis of the sample. We checked the impact of also performing linear fittings over the median values of both $\mathrm{O} / \mathrm{H}$ and $\mathrm{N} / \mathrm{O}$ in different radial bins to avoid an oversampling effect in certain radial positions in our results, but we did not find noticeable variations in our results (e.g. the average $\mathrm{O} / \mathrm{H}$ slopes changes in less than $0.01 \mathrm{dex} / R_{\mathrm{e}}$ ). In the last section, we present an analysis of the incidence in the results of the very well-known deviations from a strictly linear radial variation of the chemical abundances (i.e. decrease in central positions or flattening at the outermost H II regions). We also checked to what extent the calculation of abundances without [O II] emission lines in $\approx 30 \%$ of the H II regions can affect our results, but these are not localised in specific radial positions and/or galaxies, so the average slopes change in less than $0.01 \mathrm{dex} / R_{\mathrm{e}}$.

In addition, to rule out environmental effects between the processes that can shape the abundance gradient in galaxies, we did not consider those galaxies with some level of interaction as determined from the visual inspection of their optical images. More details on the interaction stage of each CALIFA galaxy are given in Barrera-Ballesteros et al. (2015).

This leaves the total number of analysed galaxies at 201. The list of non-interacting galaxies with at least 10 selected H II regions, with their effective radii, inclinations, resulting slopes in $\operatorname{dex} / R_{\mathrm{e}}$, and the values at the effective radius both for $\mathrm{O} / \mathrm{H}$ and N/O with their corresponding errors can be seen in Table 1.

\subsection{Properties of the resulting fittings}

In this subsection we analyse the statistical properties of the distributions of the resulting chemical abundance variations across the galactocentric de-projected distances normalised to the effective radius in the 201 CALIFA non-interacting galaxies with ten or more selected star-forming $\mathrm{H}$ II regions.

In the upper panel of Fig. 9 we show the resulting distributions and the relation between the correlation coefficients of the linear fittings to $\mathrm{O} / \mathrm{H}$ and N/O. It can be seen that most of the galaxies present a negative coefficient for $\mathrm{O} / \mathrm{H}$ and $\mathrm{N} / \mathrm{O}$, which is consistent with the classical idea that spiral galaxies have larger abundances in their inner parts and lower values at larger galactocentric distances. Accepting that the production of $\mathrm{N}$ for the metal content of these regions is mostly secondary, it is expected that we also find a negative correlation coefficient for $\mathrm{N} / \mathrm{O}$ in most of the spiral galaxies. However we also see that a nonnegligible fraction of the galaxies present a positive value of this coefficient and that a large dispersion exists between $\mathrm{O} / \mathrm{H}$ and $\mathrm{N} / \mathrm{O}$, in agreement with the large dispersion found for individual H II regions of the sample seen in Fig. 7.

The middle panel of Fig. 9 shows the distributions and relation for the $\mathrm{O} / \mathrm{H}$ and $\mathrm{N} / \mathrm{O}$ slopes of the resulting linear fittings normalised to the corresponding effective radii. Most of the galaxies present a negative gradient both for $\mathrm{O} / \mathrm{H}$ and N/O. However there is a large dispersion in the corresponding distributions 
Table 1. Analysed CALIFA galaxies with their effective radii, inclinations, and the number of $\mathrm{H}$ II regions used to calculate their $\mathrm{O} / \mathrm{H}$ and $\mathrm{N} / \mathrm{O}$ slopes and the values at the effective radius of the gradients.

\begin{tabular}{lccccccc}
\hline \hline Galaxy & $\begin{array}{c}R_{\mathrm{e}} \\
(\mathrm{kpc})\end{array}$ & $\begin{array}{c}i \\
\left({ }^{\circ}\right)\end{array}$ & H II regions & $\begin{array}{c}\alpha_{\mathrm{O} / \mathrm{H}} \\
\left(\mathrm{dex} / R_{\mathrm{e}}\right)\end{array}$ & $\begin{array}{c}12+\log (\mathrm{O} / \mathrm{H}) \\
\left(\text { at } R_{\mathrm{e}}\right)\end{array}$ & $\begin{array}{c}\alpha_{\mathrm{N} / \mathrm{O}} \\
\left(\mathrm{dex} / R_{\mathrm{e}}\right)\end{array}$ & $\begin{array}{c}\log (\mathrm{N} / \mathrm{O}) \\
\left(\text { at } R_{\mathrm{e}}\right)\end{array}$ \\
\hline ESO539-G014 & $-0.17 \pm 2.3$ & 90 & 10 & $-0.006 \pm 0.046$ & $8.602 \pm 0.083$ & $-0.172 \pm 0.070$ & $-1.420 \pm 0.120$ \\
IC 0159 & $0.19 \pm 0.2$ & 40 & 41 & $-0.053 \pm 0.013$ & $8.632 \pm 0.020$ & $-0.064 \pm 0.026$ & $-1.207 \pm 0.042$ \\
IC 0480 & $-0.02 \pm 0.5$ & 87 & 20 & $0.003 \pm 0.000$ & $8.632 \pm 0.009$ & $0.000 \pm 0.002$ & $-1.123 \pm 0.022$ \\
IC 0776 & $-0.54 \pm 0.3$ & 57 & 39 & $0.011 \pm 0.049$ & $8.574 \pm 0.062$ & $-0.001 \pm 0.026$ & $-1.444 \pm 0.034$ \\
IC 0995 & $-0.61 \pm 0.2$ & 84 & 24 & $-0.003 \pm 0.030$ & $8.619 \pm 0.051$ & $-0.155 \pm 0.026$ & $-1.337 \pm 0.042$ \\
IC 1151 & $-0.27 \pm 0.2$ & 63 & 68 & $-0.028 \pm 0.013$ & $8.636 \pm 0.019$ & $-0.117 \pm 0.012$ & $-1.208 \pm 0.020$ \\
IC 1256 & $0.24 \pm 0.2$ & 55 & 47 & $-0.105 \pm 0.017$ & $8.734 \pm 0.037$ & $-0.115 \pm 0.027$ & $-0.950 \pm 0.061$ \\
IC 1528 & $0.2 \pm 0.2$ & 72 & 70 & $-0.099 \pm 0.011$ & $8.713 \pm 0.018$ & $-0.132 \pm 0.018$ & $-1.053 \pm 0.036$ \\
IC 2095 & $-0.7 \pm 1.0$ & 90 & 14 & $-0.017 \pm 0.045$ & $8.562 \pm 0.074$ & $0.017 \pm 0.039$ & $-1.526 \pm 0.070$ \\
IC 2101 & $0.52 \pm 0.4$ & 90 & 25 & $-0.030 \pm 0.012$ & $8.666 \pm 0.025$ & $0.027 \pm 0.029$ & $-1.095 \pm 0.072$ \\
IC 2487 & $0.24 \pm 0.4$ & 84 & 24 & $-0.234 \pm 0.017$ & $8.755 \pm 0.029$ & $-0.273 \pm 0.035$ & $-1.001 \pm 0.063$ \\
IC 5309 & $0.13 \pm 0.2$ & 63 & 25 & $-0.055 \pm 0.021$ & $8.779 \pm 0.040$ & $-0.127 \pm 0.012$ & $-0.882 \pm 0.026$ \\
MCG-02-02-040 & $0.08 \pm 0.4$ & 84 & 22 & $-0.036 \pm 0.012$ & $8.691 \pm 0.029$ & $-0.062 \pm 0.023$ & $-1.023 \pm 0.054$ \\
MCG-02-03-015 & $0.32 \pm 0.7$ & 75 & 12 & $0.095 \pm 0.081$ & $8.676 \pm 0.127$ & $-0.204 \pm 0.119$ & $-0.959 \pm 0.176$ \\
MCG-02-51-004 & $0.54 \pm 1.4$ & 72 & 39 & $-0.108 \pm 0.021$ & $8.699 \pm 0.034$ & $-0.105 \pm 0.020$ & $-0.965 \pm 0.035$ \\
NGC 0001 & $0.8 \pm 0.1$ & 37 & 39 & $-0.026 \pm 0.031$ & $8.760 \pm 0.064$ & $-0.066 \pm 0.061$ & $-0.808 \pm 0.121$ \\
NGC 0023 & $1.03 \pm 0.4$ & 68 & 27 & $0.082 \pm 0.022$ & $8.715 \pm 0.039$ & $-0.040 \pm 0.060$ & $-0.842 \pm 0.112$ \\
NGC 0036 & $0.64 \pm 0.4$ & 51 & 25 & $-0.105 \pm 0.062$ & $8.747 \pm 0.094$ & $-0.211 \pm 0.079$ & $-0.931 \pm 0.125$ \\
NGC 0165 & $0.43 \pm 0.2$ & 35 & 28 & $-0.110 \pm 0.050$ & $8.776 \pm 0.078$ & $-0.048 \pm 0.069$ & $-0.877 \pm 0.121$ \\
NGC 0171 & $0.02 \pm 0.2$ & 52 & 37 & $-0.039 \pm 0.023$ & $8.691 \pm 0.044$ & $-0.130 \pm 0.028$ & $-0.758 \pm 0.054$ \\
\hline
\end{tabular}

Notes. The complete table is available at the CDS.

with standard deviations of the same order as the average slopes, which are $\alpha_{\mathrm{O} / \mathrm{H}}=-0.053 \pm 0.068$ and $\alpha_{\mathrm{N} / \mathrm{O}}=-0.104 \pm 0.096$.

To evaluate whether this large variation is a consequence of statistical fluctuations around a normal distribution, we performed a Lilliefors test. In this test the null hypothesis is that the sample comes from a Gaussian distribution and this cannot be rejected if the significance $p$ value is larger than 0.1 . The resulting $p$ values are 0.034 for $\mathrm{O} / \mathrm{H}$ and 0.001 for $\mathrm{N} / \mathrm{O}$ so, apparently, the queue of flat and positive slopes seen in both distributions are not the consequence of a statistical fluctuation.

Although these results confirm the trend towards a negative radial gradient both for $\mathrm{O} / \mathrm{H}$ and $\mathrm{N} / \mathrm{O}$ (e.g. Sánchez et al. 2014; Pilyugin et al. 2004), there is a non-negligible fraction of objects that present a flat or even a positive gradient that is already observed in the sample of CALIFA galaxies by Sánchez et al. (2014). On the contrary, no objects with inverted $\mathrm{O} / \mathrm{H}$ or N/O gradients are found by Pilyugin et al. (2004), but the sample analysed there is much smaller. The fraction of these positive gradients are $\approx 19 \%$ for $\mathrm{O} / \mathrm{H}$ and $10 \%$ for $\mathrm{N} / \mathrm{O}$. However these fractions are reduced to $10 \%$ and $4 \%$, respectively, when we only consider those galaxies with a positive slope within the error. The number of galaxies and the average properties of the different subsamples as a function of the $\mathrm{O} / \mathrm{H}$ and $\mathrm{N} / \mathrm{O}$ slopes are shown in Table 2.

Interestingly, the average slope for $\mathrm{N} / \mathrm{O}$ is more pronounced than for $\mathrm{O} / \mathrm{H}$. At same time, the number of objects with an inverted $\mathrm{N} / \mathrm{O}$ gradient is lower than for $\mathrm{O} / \mathrm{H}$. These differences could be caused by two factors. Firstly, N/O is more precise than $\mathrm{O} / \mathrm{H}$ because the range of variation of $\mathrm{N} / \mathrm{O}$ for the $\mathrm{H}$ II regions in this sample is much larger with a lower associated mean error (see Fig. 7, i.e. the average slope of $\mathrm{O} / \mathrm{H}$ represents a $5 \%$ of the $\mathrm{O} / \mathrm{H}$ range for the CALIFA $\mathrm{H}$ II regions and a $2.5 \%$ for $\mathrm{N} / \mathrm{O}$ ). Secondly, N/O tends to be unaffected by processes related to hydrodynamical effects caused by the interchange of (un-)processed material between different parts of the galaxies or even to the surrounding IGM. This underlines once again the importance of the determination of N/O to calculate chemical abundance gradients.

This N/O dependence can partially explain the lower average slope in this work as compared with Sánchez et al. (2014) and Pilyugin et al. (2004). By using the $\mathrm{O} / \mathrm{H}$ abundances derived by $\mathrm{HCM}$, but without a previous N/O determination (i.e. using a grid of models assuming a tight $\mathrm{O} / \mathrm{H}-\mathrm{N} / \mathrm{O}$ relation as shown by the cyan line in Fig. 7), the resulting average slope for the same sample of galaxies is $\alpha_{\mathrm{O} / \mathrm{H}}=-0.069 \mathrm{dex} / R_{\mathrm{e}}$. The difference between the $\mathrm{O} / \mathrm{H}$ slope, which is derived in a galaxy using [N II] lines when we consider a previous N/O calculation, depends on the resulting N/O slope, as can be seen in left panel of Fig. 10. This difference is negative for galaxies with a flat or inverted N/O gradient and positive for negative N/O slopes (i.e. when we calculate $\mathrm{N} / \mathrm{O}$, the resulting $\mathrm{O} / \mathrm{H}$ slopes are flatter in those galaxies with a steeper $\mathrm{N} / \mathrm{O}$ negative gradient). On average, the mean $\mathrm{O} / \mathrm{H}$ slope is 0.02 dex flatter when we consider a previous N/O estimation in the space of the grid of models for this sample. The difference is very small if we compare the $\mathrm{O} / \mathrm{H}$ slopes that are derived using this constrained grid of models with the $\mathrm{O} / \mathrm{H}$ slopes derived using a strong-line method that does not consider any N/O dependence. In right panel of Fig. 10 we show a comparison with the slopes obtained from the O3N2 parameter as calibrated by Marino et al. (2013), and the residuals have an average lower than $0.01 \mathrm{dex} / R_{\mathrm{e}}$.

On the other hand, the differences between the behaviours of $\mathrm{O} / \mathrm{H}$ and $\mathrm{N} / \mathrm{O}$ radial variations are also evidenced by the not very high correlation between the slopes (Spearman's coefficient, $\rho_{\mathrm{s}}=0.39$ ). Indeed, as it can be seen in Table 2, the average $\mathrm{N} / \mathrm{O}$ slope for those galaxies with a positive $\mathrm{O} / \mathrm{H}$ gradient is negative, although it is flatter than for the whole sample. Similarly, the average $\mathrm{O} / \mathrm{H}$ slope for those galaxies with a positive $\mathrm{N} / \mathrm{O}$ gradient is negative although, again, with a flatter value as compared with all galaxies. The lack of a perfect correlation between $\mathrm{O} / \mathrm{H}$ 

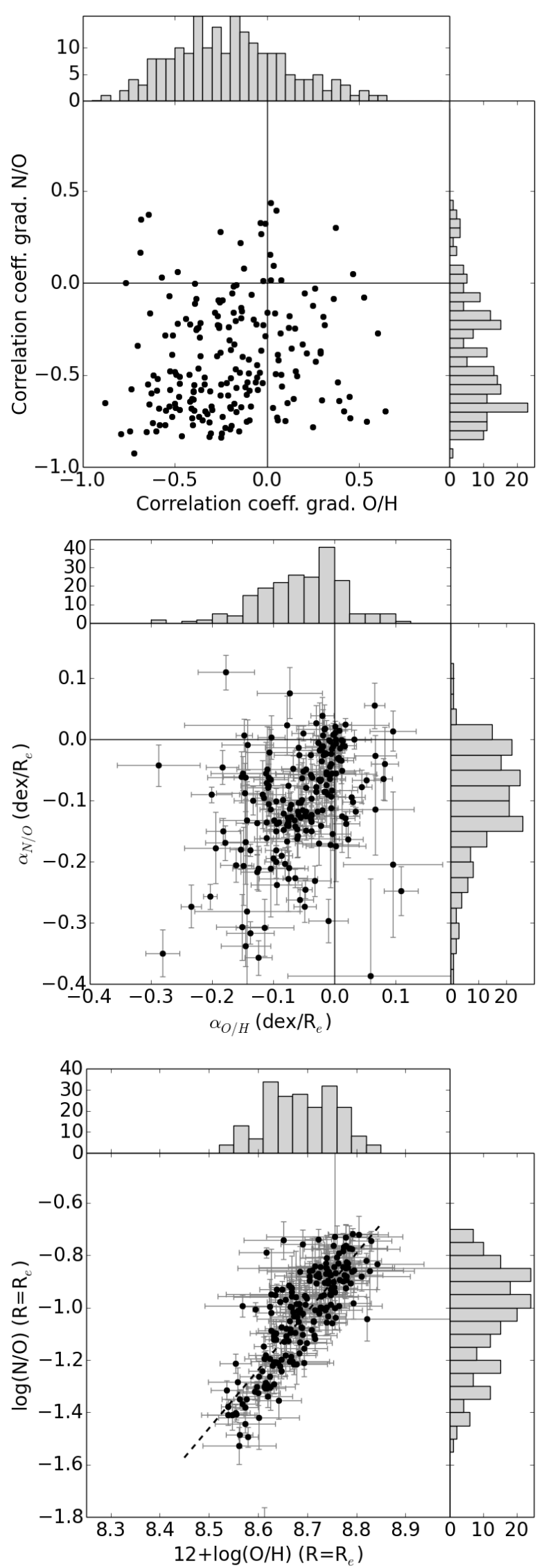

Fig. 9. Histograms and relations of the properties derived from the linear fittings through the radial scale both for $\mathrm{O} / \mathrm{H}$ and $\mathrm{N} / \mathrm{O}$ in those galaxy discs of the CALIFA sample with enough star-forming $\mathrm{H}$ II regions. From top to bottom: correlation coefficient, slope of the gradient, and predicted value at the effective radius of the galaxy. and N/O gradients is illustrated in Fig. 11, where four different combinations of gradient trends for $\mathrm{O} / \mathrm{H}$ and $\mathrm{N} / \mathrm{O}$ are shown.

Finally, the lower panel of Fig. 9 shows the distributions and scatter plot for the $\mathrm{O} / \mathrm{H}$ and $\mathrm{N} / \mathrm{O}$ values at the effective radius as calculated from the robust error-weighted linear fittings. The average values are listed for all galaxies and as a function of the $\mathrm{O} / \mathrm{H}$ and $\mathrm{N} / \mathrm{O}$ slopes in Table 2. Contrary to the relation between abundance ratios shown in Fig. 7 for individual $\mathrm{H}$ II regions, the dispersion is very low and there is a high correlation coefficient ( $\rho_{\mathrm{s}}=0.80$, while for individual H II regions is 0.38 ) between the characteristic $\mathrm{O} / \mathrm{H}$ and $\mathrm{N} / \mathrm{O}$ values. A linear fitting to these points yields the following relation:

$\log (\mathrm{N} / \mathrm{O})=-20.39+2.23 \cdot[12+\log (\mathrm{O} / \mathrm{H})]$,

with a standard deviation of the residuals of 0.12 dex. This linear fitting is also plotted in the lower panel of Fig. 9.

This very good correlation between $\mathrm{O} / \mathrm{H}$ and $\mathrm{N} / \mathrm{O}$ values at the effective radius, in contrast to the same relation for individual $\mathrm{H}$ II regions or to the slopes of the same gradients, could be the consequence that the typical expected values of $Z$ are not so sensitive to possible internal variations across the discs, but mostly depend on other integrated properties of the galaxies. This idea was already shown by Pilyugin et al. (2004), where a characteristic abundance value for spiral galaxies correlates much better with some of the properties of the discs. According to these authors, this value corresponds to the $Z$ predicted by the linear fitting at 0.4 times the isophotal radius $\left(R_{25}\right)$. In our sample of selected CALIFA galaxies, the galactocentric distance $0.4 \times R_{25}$ corresponds on average to $\approx 2$ times the effective radius. But, in what follows, we consider the values at the effective radius as typical values of the metal content of the galaxies studied here, to study possible correlations between the characteristic $\mathrm{O} / \mathrm{H}$ and $\mathrm{N} / \mathrm{O}$ at the effective radius and to study the relation between these abundances and other integrated properties of the galaxies. This correlation between integrated properties and the fitted radial structure of the spiral galaxies studied in the CALIFA survey has been also observed in the stellar parameters by González Delgado et al. (2015).

\section{Correlation with integrated galactic properties}

\subsection{Relation with luminosity and stellar mass}

In this subsection we study to what extent is there a statistical dependence of the slopes or the characteristic abundance ratios on the total luminosity and the stellar mass of the 201 CALIFA galaxies with a derivations of the $\mathrm{O} / \mathrm{H}$ and $\mathrm{N} / \mathrm{O}$ radial variations.

In Fig. 12 we show the derived slopes and the characteristic values both for $\mathrm{O} / \mathrm{H}$ and $\mathrm{N} / \mathrm{O}$ along with the running medians for bins of 25 elements as a function of the integrated absolute magnitude in the SDSS filter $g$ as derived by Walcher et al. (2014) using growth curves. As can be seen, there is a slight trend towards flatter gradients for both $\mathrm{O} / \mathrm{H}$ and $\mathrm{N} / \mathrm{O}$ for lower luminosities. This is more evident in the case of N/O, although, in both cases, the errors prevent us from being totally confident with this result. For the less luminous galaxies of this sample $\left(M_{\mathrm{g}}>-19.5\right)$ the mean slope is $-0.014 \mathrm{dex} / R_{\mathrm{e}}$ for $\mathrm{O} / \mathrm{H}$ and $-0.055 \mathrm{dex} / R_{\mathrm{e}}$ for $\mathrm{N} / \mathrm{O}$, while for the more luminous $\left(M_{\mathrm{g}}<-20.25\right)$ the mean slopes are -0.067 for $\mathrm{O} / \mathrm{H}$ and $-0.118 \mathrm{dex} / R_{\mathrm{e}}$ for $\mathrm{N} / \mathrm{O}$.

Very similar results are found when we look at the relation with the total stellar mass, which is shown in Fig. 13 both for $\mathrm{O} / \mathrm{H}$ and $\mathrm{N} / \mathrm{O}$. The stellar mass derivations for this sample are described in Sánchez et al. (2013). The average slopes for galaxies with $\log \left(M / M_{\odot}\right)<9.5$ are less prominent for both $\mathrm{O} / \mathrm{H}$ 

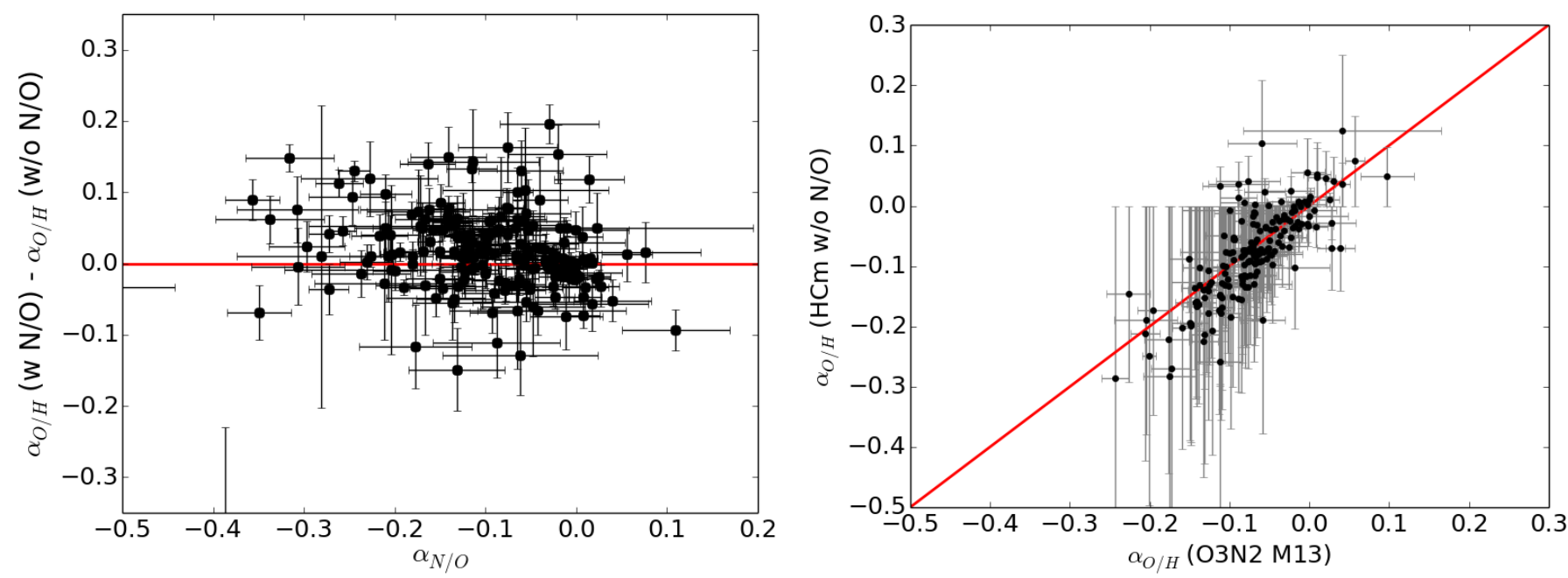

Fig. 10. At left, difference as a function of the N/O slope of the $\mathrm{O} / \mathrm{H}$ slopes calculated with a previous N/O calculation and the same slopes calculated assuming a given $\mathrm{O} / \mathrm{H}-\mathrm{N} / \mathrm{O}$ relation for all the analysed CALIFA galaxies. The red solid line represents the zero value. At right, comparison between the $\mathrm{O} / \mathrm{H}$ slopes calculated using this last approach with the same slopes calculated using the O3N2 parameter as calibrated by Marino et al. (2013). The red solid line represents the 1:1 relation. All slopes are expressed in dex $/ R_{\mathrm{e}}$.
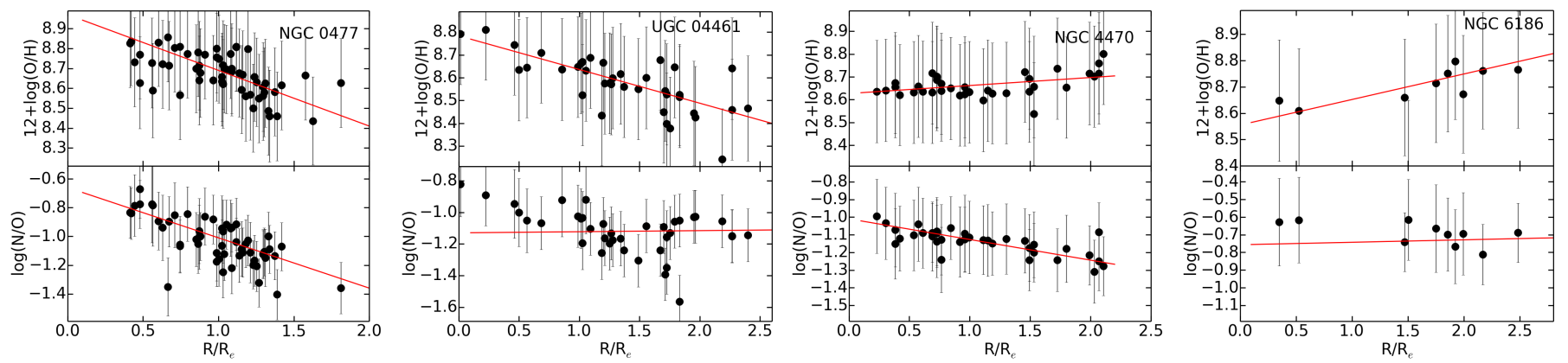

Fig. 11. Four examples of different combinations of slopes in $\mathrm{O} / \mathrm{H}$ and $\mathrm{N} / \mathrm{O}$ in the sample of the CALIFA galaxies. From left to right: NGC 0477 presents negative gradients both for $\mathrm{O} / \mathrm{H}$ and $\mathrm{N} / \mathrm{O}$, UGC 04461 shows a negative gradient of $\mathrm{O} / \mathrm{H}$, but the N/O gradient is flat. NGC 4470 has positive $\mathrm{O} / \mathrm{H}$ gradient and a negative N/O gradient and, finally, NGC 6186 shows a positive gradient both for $\mathrm{O} / \mathrm{H}$ and N/O.

Table 2. Average slopes and values at the effective radius for the linear fittings of $\mathrm{O} / \mathrm{H}$ and $\mathrm{N} / \mathrm{O}$ in the analysed CALIFA galaxies grouped by categories depending on the sign of the slopes.

\begin{tabular}{lcccccc}
\hline \hline & Number & $\%$ & $\begin{array}{c}\alpha_{\mathrm{O} / \mathrm{H}} \\
\left(\mathrm{dex} / R_{\mathrm{e}}\right)\end{array}$ & $\begin{array}{c}12+\log (\mathrm{O} / \mathrm{H}) \\
\left(\text { at } R_{\mathrm{e}}\right)\end{array}$ & $\begin{array}{c}\alpha_{\mathrm{N} / \mathrm{O}} \\
\left(\mathrm{dex} / R_{\mathrm{e}}\right)\end{array}$ & $\begin{array}{c}\log (\mathrm{N} / \mathrm{O}) \\
\left(\text { at } R_{\mathrm{e}}\right)\end{array}$ \\
\hline All & 201 & 100 & $-0.053 \pm 0.068$ & $8.688 \pm 0.070$ & $-0.104 \pm 0.096$ & $-1.041 \pm 0.198$ \\
$\alpha_{\mathrm{O} / \mathrm{H}}<0$ & 162 & 80.6 & $-0.073 \pm 0.057$ & $8.697 \pm 0.068$ & $-0.110 \pm 0.086$ & $-1.026 \pm 0.181$ \\
$\alpha_{\mathrm{O} / \mathrm{H}}<0(1 \sigma)$ & 130 & 64.7 & $-0.088 \pm 0.054$ & $8.702 \pm 0.066$ & $-0.121 \pm 0.087$ & $-1.012 \pm 0.174$ \\
$\alpha_{\mathrm{O} / \mathrm{H}}>0$ & 39 & 19.4 & $+0.032 \pm 0.032$ & $8.652 \pm 0.069$ & $-0.079 \pm 0.125$ & $-1.104 \pm 0.250$ \\
$\alpha_{\mathrm{O} / \mathrm{H}}>0(1 \sigma)$ & 20 & 10.0 & $+0.048 \pm 0.033$ & $8.652 \pm 0.067$ & $-0.062 \pm 0.077$ & $-1.041 \pm 0.216$ \\
$\alpha_{\mathrm{N} / \mathrm{O}}<0$ & 186 & 92.5 & $-0.056 \pm 0.068$ & $8.690 \pm 0.071$ & $-0.118 \pm 0.090$ & $-1.036 \pm 0.198$ \\
$\alpha_{\mathrm{N} / \mathrm{O}}<0(1 \sigma)$ & 156 & 77.6 & $-0.062 \pm 0.068$ & $8.695 \pm 0.069$ & $-0.133 \pm 0.088$ & $-1.030 \pm 0.194$ \\
$\alpha_{\mathrm{N} / \mathrm{O}}>0$ & 20 & 10.0 & $-0.027 \pm 0.065$ & $8.678 \pm 0066$ & $+0.025 \pm 0.027$ & $-1.075 \pm 0.205$ \\
$\alpha_{\mathrm{N} / \mathrm{O}}>0(1 \sigma)$ & 9 & 4.5 & $-0.021 \pm 0.061$ & $8.674 \pm 0.062$ & $+0.041 \pm 0.033$ & $-1.107 \pm 0.190$ \\
\hline
\end{tabular}

$\left(-0.019 \mathrm{dex} / R_{\mathrm{e}}\right)$ and N/O $\left(-0.061 \mathrm{dex} / R_{\mathrm{e}}\right)$ than for the rest of the sample. However, no clear trend is seen in this case for the high-mass galaxies. When we restrict the sample of galaxies to those with an inclination that is lower than $70^{\circ}(113 \mathrm{ob}-$ jects), we still see the same trend towards sensibly shallower slopes for low luminosity/mass objects (i.e. for $M_{\mathrm{g}}>-19.5$, $\alpha_{\mathrm{O} / \mathrm{H}}=-0.028 \mathrm{dex} / R_{\mathrm{e}}$ and $\left.\alpha_{\mathrm{N} / \mathrm{O}}=-0.059 \mathrm{dex} / R_{\mathrm{e}}\right)$. Other works (e.g. Sánchez et al. 2014; Ho et al. 2015) have not found any clear correlation between the average slope of the $\mathrm{O} / \mathrm{H}$ gradients, once normalised by the effective radius, with the total luminosity or the total stellar mass. Our results point to a trend towards flatter slopes for less luminous and less massive galaxies, but it is necessary to improve the statistical significance of the results to confirm this trend.

In the lower panels of Fig. 12, in the case of the integrated absolute $g$ luminosity, and in Fig. 13, for the total stellar mass, we can see the respective relations with the obtained values of $\mathrm{O} / \mathrm{H}$ and $\mathrm{N} / \mathrm{O}$ at the effective radii. As can be seen, there are tight and very low dispersion relations, confirming for this sample the known connections between the total luminosity and/or stellar 

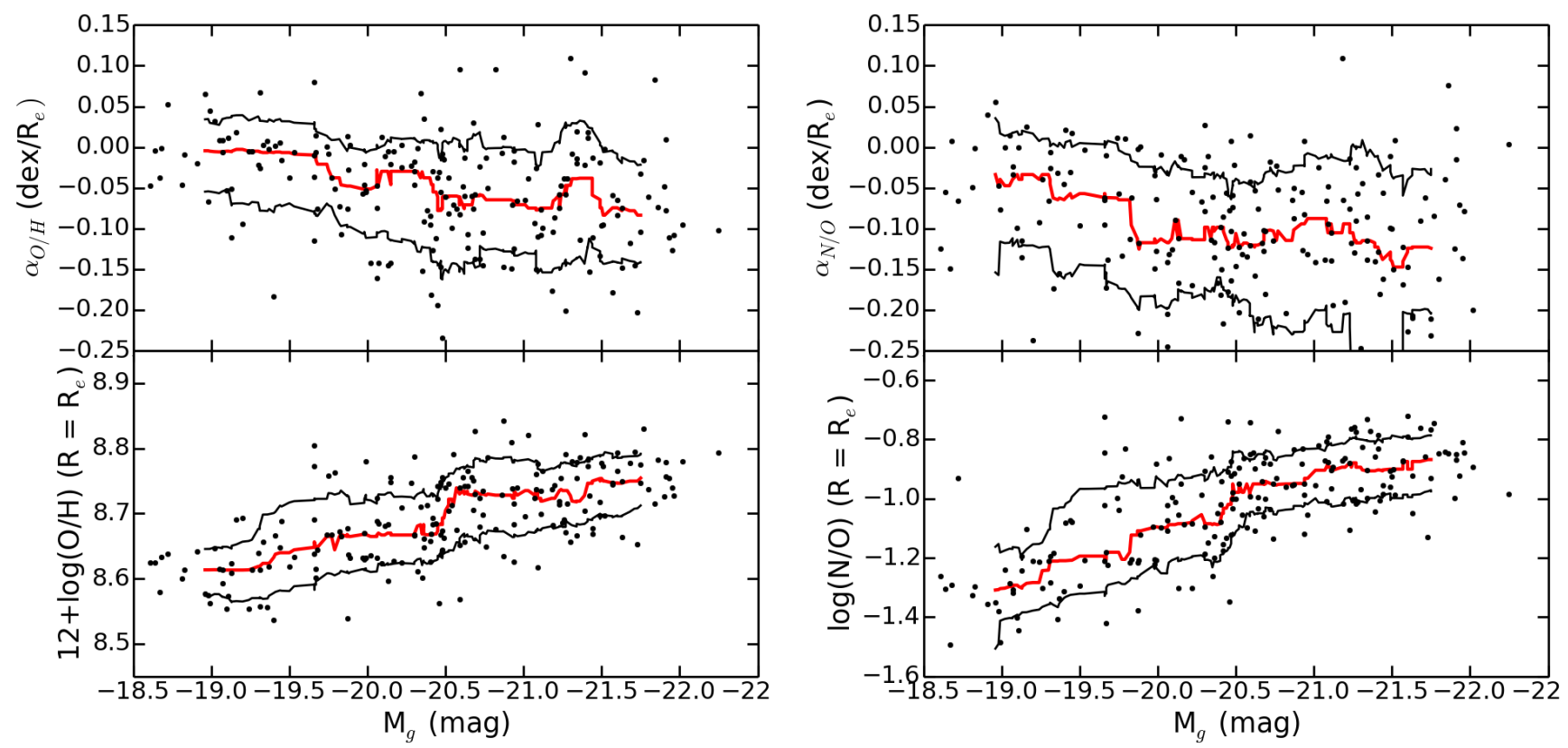

Fig. 12. Relation between the absolute luminosity in the $g$ band and the derived slopes and characteristic values at the effective radius for $\mathrm{O} / \mathrm{H}$ (left panels) and N/O (right panels). The red solid line represents the running median for bins of 25 objects, and the black solid lines the $1 \sigma$ above and below the averages in the same bins.
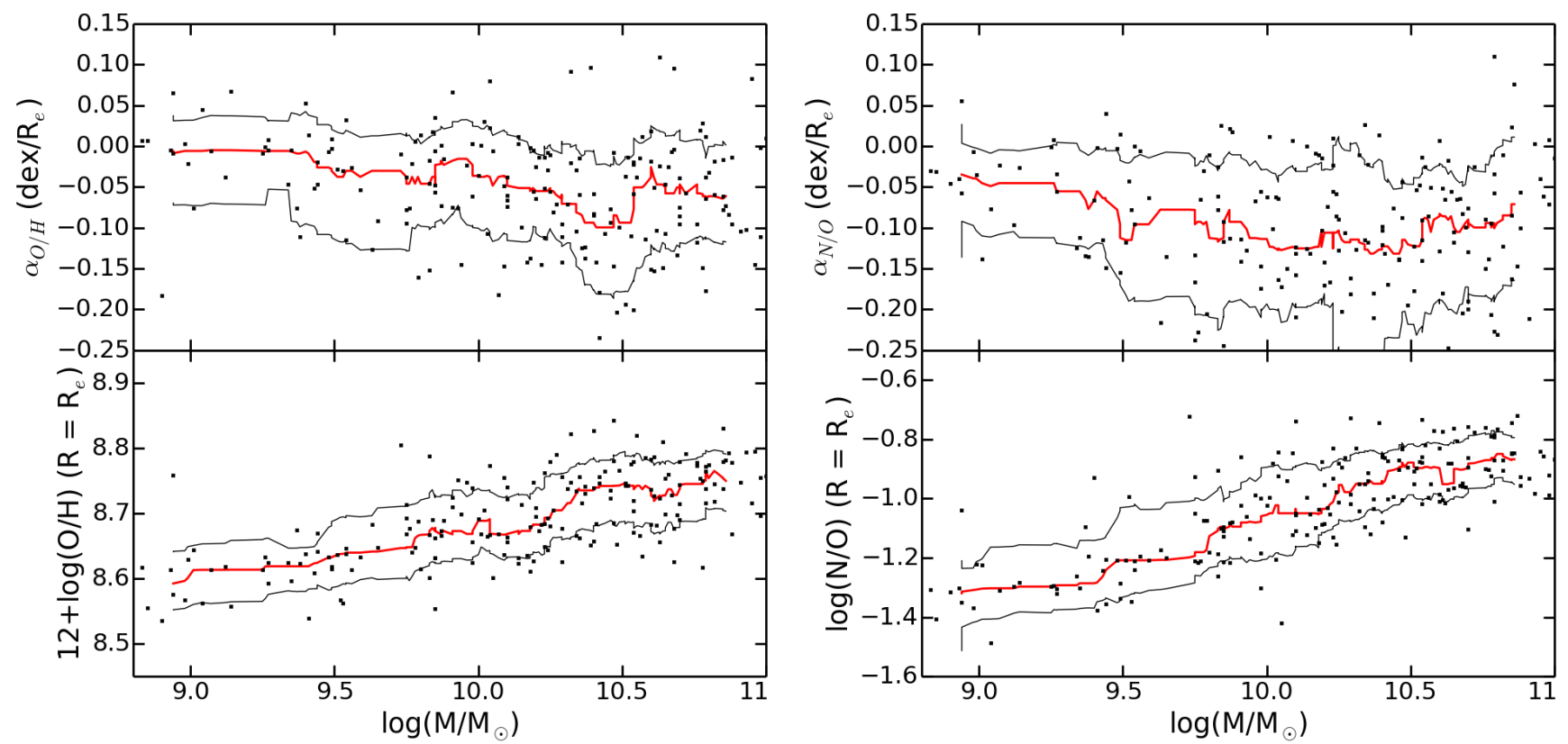

Fig. 13. Relation between the total stellar mass and the derived slopes and characteristic values at the effective radius for $\mathrm{O} / \mathrm{H}$ (left panels) and N/O (right panels). The red and black solid lines have the same meaning as in Fig. 12.

mass of a galaxy with $Z$ content (e.g. Lamareille et al. 2004; Tremonti et al. 2004) and with N/O (Pérez-Montero \& Contini 2009; Pérez-Montero et al. 2013). In the case of $\mathrm{O} / \mathrm{H}$ this result confirms that obtained by Pilyugin et al. (2004) who find a tighter correlation with luminosity, as a proxy of the stellar mass, and with rotation velocity, as a proxy of the dynamical mass, when a characteristic oxygen abundance value at a specific radial position from a fitting is assumed. This characteristic value was situated at 0.4 times the isophotal radius, instead of using an integrated value of $Z$.

In our case a quadratic relation can be fitted between the total stellar masses and the $\mathrm{O} / \mathrm{H}$ values at the effective radius

$y_{1}=8.1994+0.0 .0168 \cdot x-0.0031 \cdot x^{2}$, where $x$ is $\log \left(M / M_{\odot}\right)$ and $y_{1}$ is $\mathrm{O} / \mathrm{H}$ in terms of $12+\log (\mathrm{O} / \mathrm{H})$. This fitting only has a standard deviation of the residuals for the 201 studied galaxies of 0.03 dex, which is much lower than the uncertainty associated with the $\mathrm{O} / \mathrm{H}$ derivation and the $\mathrm{O} / \mathrm{H}$ characteristic values in each galaxy.

A similar fitting for $\mathrm{N} / \mathrm{O}$ at the effective radius yields

$y_{2}=-8.0611+1.1581 \cdot x-0.0456 \cot x^{2}$,

where $x$ is $\log \left(M / M_{\odot}\right)$ and $y_{2}$ is $\mathrm{N} / \mathrm{O}$ in terms of $\log (\mathrm{N} / \mathrm{O})$. For this fitting the standard deviation of the residuals is also of 0.03 dex, which is much lower than the error associated with the $\mathrm{N} / \mathrm{O}$ derivation and the characteristic $\mathrm{N} / \mathrm{O}$ values. The quadratic fittings found in the relations between the total stellar mass and 

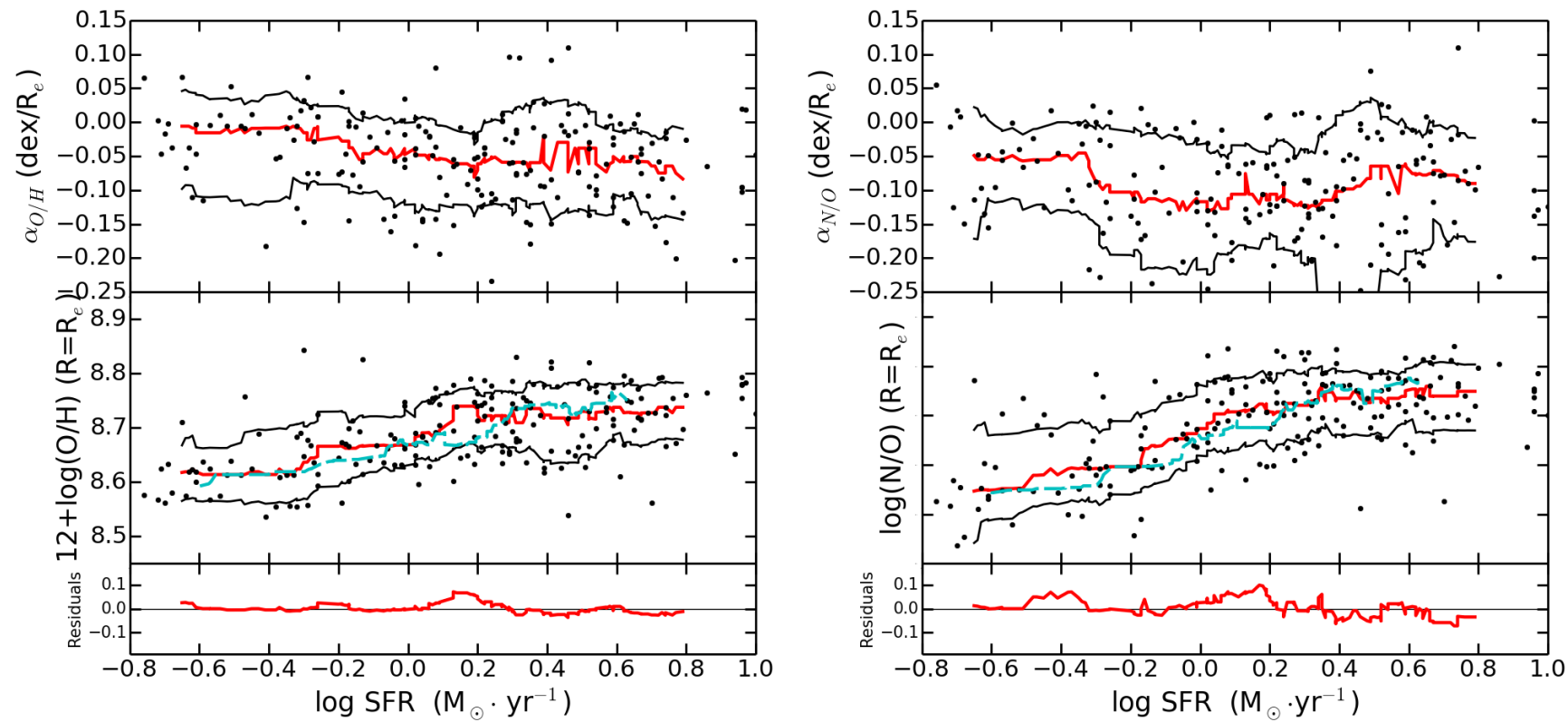

Fig. 14. Relation between the integrated SFR and the derived slopes and characteristic values at the effective radius for $\mathrm{O} / \mathrm{H}$ (left panels) and N/O (right panels). The red and black solid lines in the upper and middle panels have the same meaning as in Fig. 12. In the middle panels, the dashed cyan line represents the running median assuming the observed relation between the SFR and stellar mass. In the lower panel, the residuals are indicated in red and the running medians are indicated in cyan.

both the $\mathrm{O} / \mathrm{H}$ and N/O characteristic values confirm the trend already observed (e.g. Tremonti et al. 2004; Pilyugin et al. 2007) of a flattening of the MZR at high masses and luminosities.

\subsection{Relation with present-day star formation and colour}

Regarding the relation between the metal content of a galaxy and its total SFR, it has been suggested, for certain samples of galaxies, that, using the integrated emission of star-forming galaxies, there is a dependence between $Z$ and SFR. This dependence points towards lower $Z$ values for higher SFRs in a specific mass bin, thereby reducing the dispersion of the MZR in the so-called fundamental metallicity relation (FMR; e.g. Mannucci et al. 2010; Lara-López et al. 2010). According to Pérez-Montero et al. (2013) from the analysis of SDSS data, the SFR dependence is not observed with N/O, so this could be indicative that the SFR- $Z$ for each mass bin is due to the presence of inflows of metal-poor gas and/or outflows of enriched gas, that do not affect N/O, but can considerably alter both the SFR and $Z$.

It is thus interesting to examine whether the slopes or the characteristic values of the chemical abundances calculated for the sample of CALIFA galaxies depend on their integrated SFR. The integrated SFR were calculated using the extinctioncorrected integrated $\mathrm{H} \alpha$ fluxes following the same procedure as described in Sánchez et al. (2013).

In the upper panels of Fig. 14 we see the derived $\mathrm{O} / \mathrm{H}$ and $\mathrm{N} / \mathrm{O}$ slopes, with their corresponding running medians, as a function of $\log$ SFR. As in the case of stellar mass, there is a very slight, not statistically significant, difference between the $\mathrm{O} / \mathrm{H}$ slopes of low and high SFR (i.e. $\alpha_{\mathrm{O} / \mathrm{H}}=-0.043 \mathrm{dex} / R_{\mathrm{e}}$ for SFRs lower than the mean value, $10^{0.2} M_{\odot} / \mathrm{yr}$, and -0.062 for higher SFRs). A similar trend is obtained for $\mathrm{N} / \mathrm{O}$, but the difference between the two bins is lower than $0.01 \mathrm{dex} / R_{\mathrm{e}}$.

Much more clearly, in the middle panels of the same figures, we see that there is a trend both for $\mathrm{O} / \mathrm{H}$ and $\mathrm{N} / \mathrm{O}$ to find higher characteristic values at the effective radius for galaxies with a higher integrated SFR. To check whether this relation is contrary to the FMR, we examine the relation between SFR and the stellar mass. In the left panel of Fig. 15 we show this relation for the sample of CALIFA galaxies selected for the analysis of radial gradients. As in the case of the galaxies of the main sequence of star formation, as shown in Brinchmann et al. (2004), the SFR correlates with the stellar mass of the galaxies, as expected from the fact that larger galaxies have larger gas repositories. Taking the mass-SFR relation that is obtained from the corresponding running median for this sample, we used the observed MZR and MNOR plotted in Fig. 13 to compare the resulting SFR-O/H or SFR-N/O with the same observed relations. As can be seen in the lower panels of Fig. 14, the residuals between the SFR-O/H (at left) and SFR-N/O (at right) and the same relation considering the observed M-SFR relation are very small. The standard deviations of these residuals are 0.03 dex for both plots with no mean deviation at any SFR. This M-SFR relation also explains the slight slope difference observed for $\mathrm{O} / \mathrm{H}$, as low-mass galaxies have, on average, slightly flatter slopes.

We can therefore conclude that no additional dependence between $\mathrm{O} / \mathrm{H}$ or $\mathrm{N} / \mathrm{O}$ and SFR is observed for this sample of galaxies contrary to the results pointing to the existence of the FMR. This agrees with the results in Sánchez et al. (2013) for individual CALIFA H II regions, for which no additional relation between $\mathrm{O} / \mathrm{H}$ and SFR is found, supporting a scenario of gas recycling faster than gas accretion from inflows or mass loss by outflows.

Although a certain relation between $\mathrm{O} / \mathrm{H}$ (or N/O) and the SFR is observed for individual $\mathrm{H}$ II regions, this is again a consequence of the M-SFR relation. In Fig. 16, a certain correlation between the $\mathrm{O} / \mathrm{H}$ of the individual $\mathrm{H}$ II regions and their $\mathrm{H} \alpha \mathrm{lu}-$ minosity $\left(\rho_{\mathrm{s}}=0.17\right)$ can be seen, which is even clearer for $\mathrm{N} / \mathrm{O}$ $\left(\rho_{\mathrm{s}}=0.42\right)$. These correlations can be explained as due to the stellar mass of the galaxies hosting the H II regions. The average $L(\mathrm{H} \alpha)$ is lower for the less massive galaxies than for the more massive galaxies. The mean $L(\mathrm{H} \alpha)=10^{38.89} \mathrm{erg} / \mathrm{s}$ for $M_{*}<$ $10^{10.22} M_{\odot}$ and $10^{39.43}$ for higher masses, as $M=10^{10.22} M_{\odot}$ is 

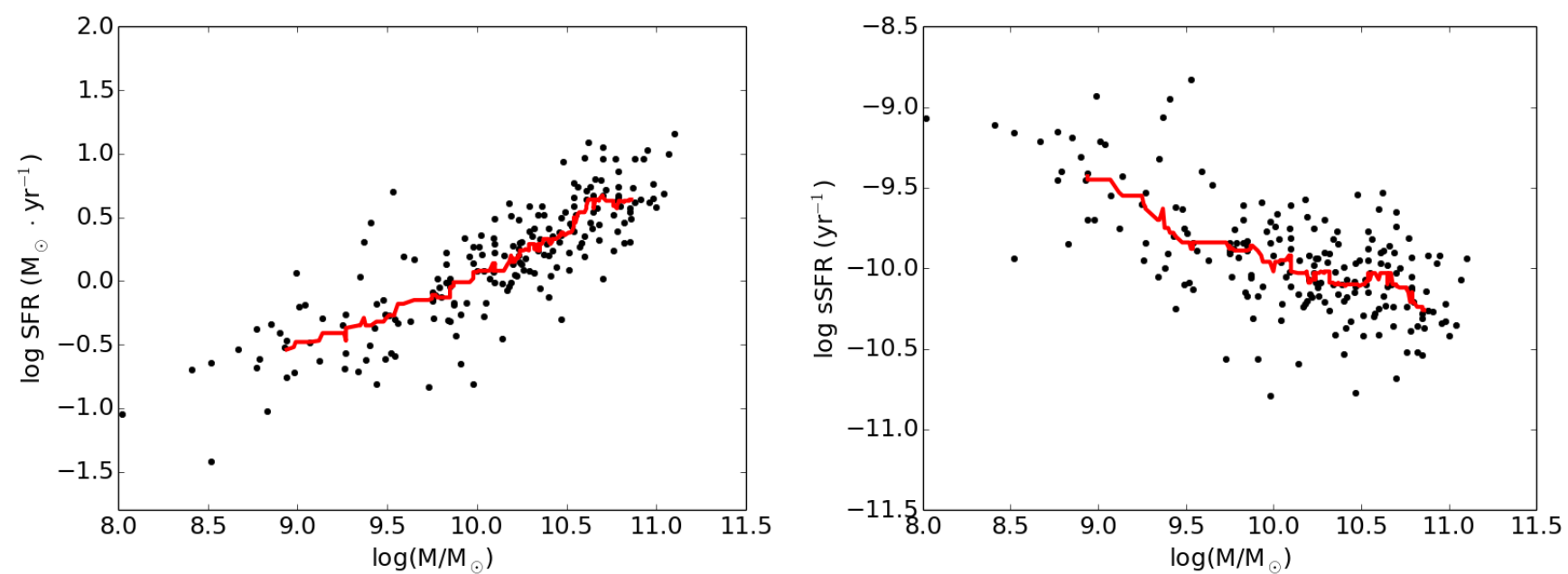

Fig. 15. Relation between the total stellar mass and the present-day integrated SFR (at left) and the sSFR (at right) for the 201 selected spiral non-interacting CALIFA galaxies. The red solid lines represent the running median for bins of 25 objects.
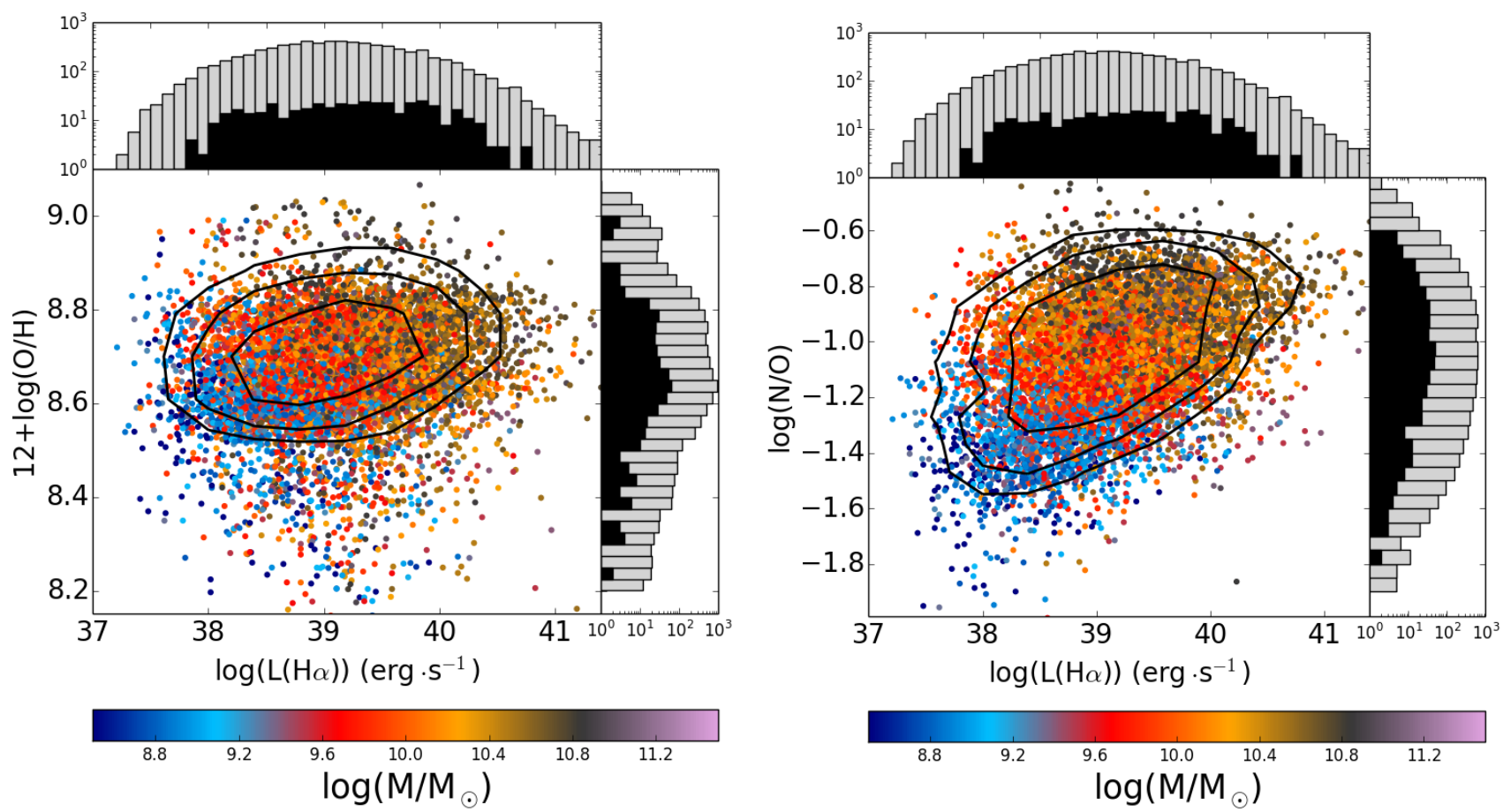

Fig. 16. Relation between the $\mathrm{H} \alpha$ luminosity and $\mathrm{O} / \mathrm{H}$, at left, and $\mathrm{N} / \mathrm{O}$, at right, for the selected $\mathrm{H}$ II regions of the galaxies for which a linear gradient was calculated. The colour encodes the total stellar mass of the galaxy where each H II region is situated. The histograms show in logarithmic scales in all axes the distribution for all objects represented in the plot (white bars) and for those H II regions in a galaxy with an inverted gradient (black bars). Colours indicate the density of points and solid lines represent the $1 \sigma, 2 \sigma$, and $3 \sigma$ contours.

the median stellar mass of the 201 galaxies with an estimate of the abundance gradient.

Very similar results are obtained when we analyse the dependence between the slopes of the gradients or the characteristic abundance values on the specific star formation rate ( $S S F R$ $=S F R / M)$, which can be considered an indicator of the ratio between present-day and past SFR. There is also evidence in the literature that specific mass bin galaxies with higher sSFR have on average lower $Z$ (e.g. Ellison et al. 2008). The resulting median slopes and characteristic values can be seen in Fig. 17.

As in the case of the mass or the SFR, a slight trend towards flatter $\mathrm{O} / \mathrm{H}$ and $\mathrm{N} / \mathrm{O}$ gradients for galaxies with a lower sSFR is found (i.e. $\alpha_{\mathrm{O} / \mathrm{H}}=-0.029 \mathrm{dex} / R_{\mathrm{e}}\left(\alpha_{\mathrm{N} / \mathrm{O}}=-0.085\right)$ ) for the bin of sSFR that is lower than the median $\left(10^{-9.97} \mathrm{yr}^{-1}\right.$ and $\alpha_{\mathrm{O} / \mathrm{H}}=$ $\left.-0.059 \mathrm{dex} / R_{\mathrm{e}}\left(\alpha_{\mathrm{N} / \mathrm{O}}=-0.105\right)\right)$ for the upper sSFR bin.

Regarding characteristic values, a clear trend towards lower values both for $\mathrm{O} / \mathrm{H}$ and $\mathrm{N} / \mathrm{O}$ for higher values of sSFR is seen in the middle panels of the same figure. However, as in the case of SFR, we see that this relation is owing to the previously studied MZR in combination with the relation between mass and sSFR observed in right panel of Fig. 15. This sample of galaxies, again, shows a clear relation in this plot as the SSFR is lower for galaxies of higher stellar mass. By comparing, in the lower panels of Fig. 14, the relations $\mathrm{O} / \mathrm{H}-\mathrm{s} S F R$ or $\mathrm{N} / \mathrm{O}-\mathrm{s} S F R$ with the running medians of the MZR using the $M-\mathrm{S} S F R$ relation, we find that the standard deviation of the residuals is lower than 0.02 dex in both cases. 

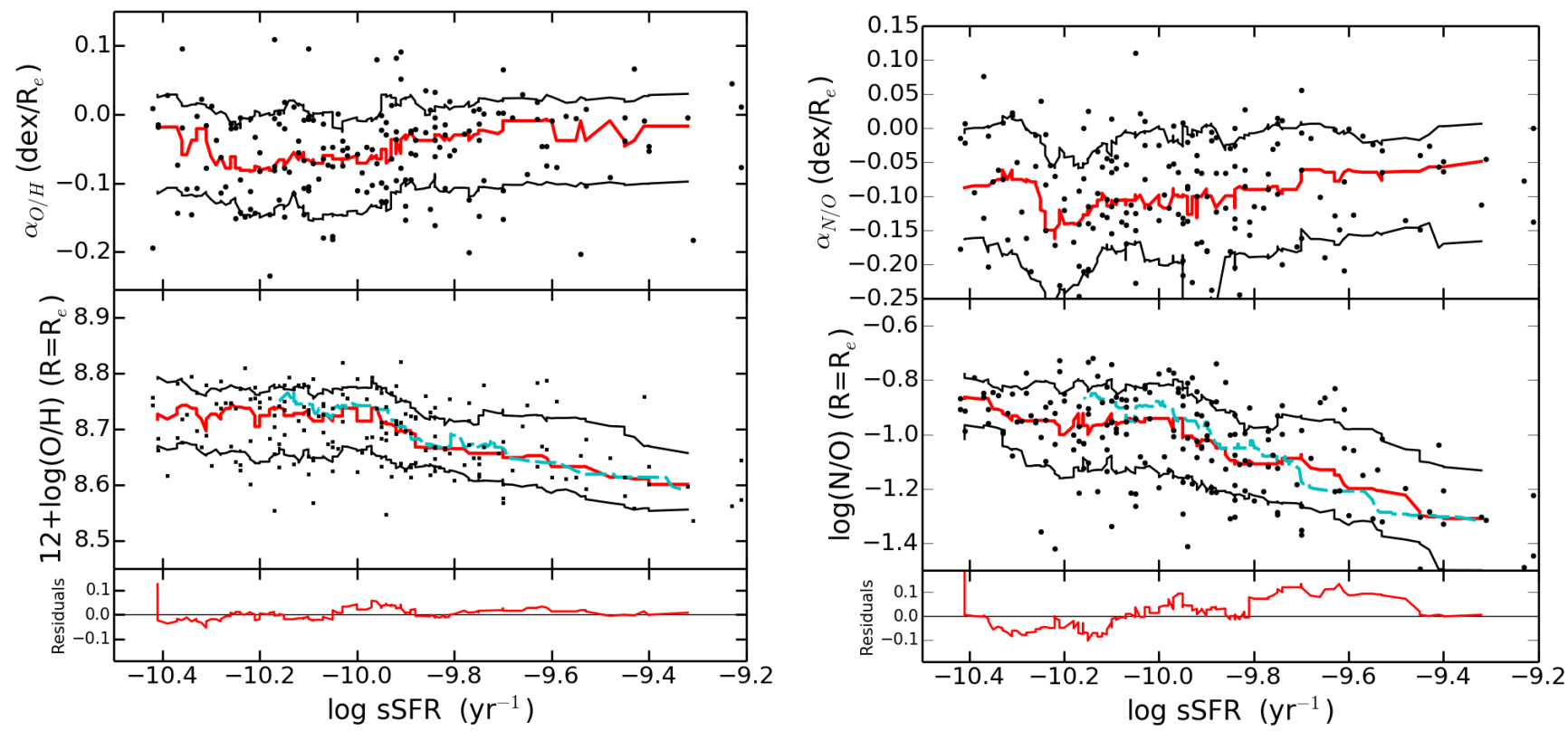

Fig. 17. Relation between the integrated sSFR and the derived slopes and characteristic values at the effective radius for O/H (left panels) and N/O (right panels). The red and black solid lines in the upper and middle panels have the same meaning as in Fig. 12. In the middle panels, the dashed cyan line represents the running median assuming the observed relation between the SFR and stellar mass. In the lower panel, the residuals are indicated in red and running medians are indicated in cyan.
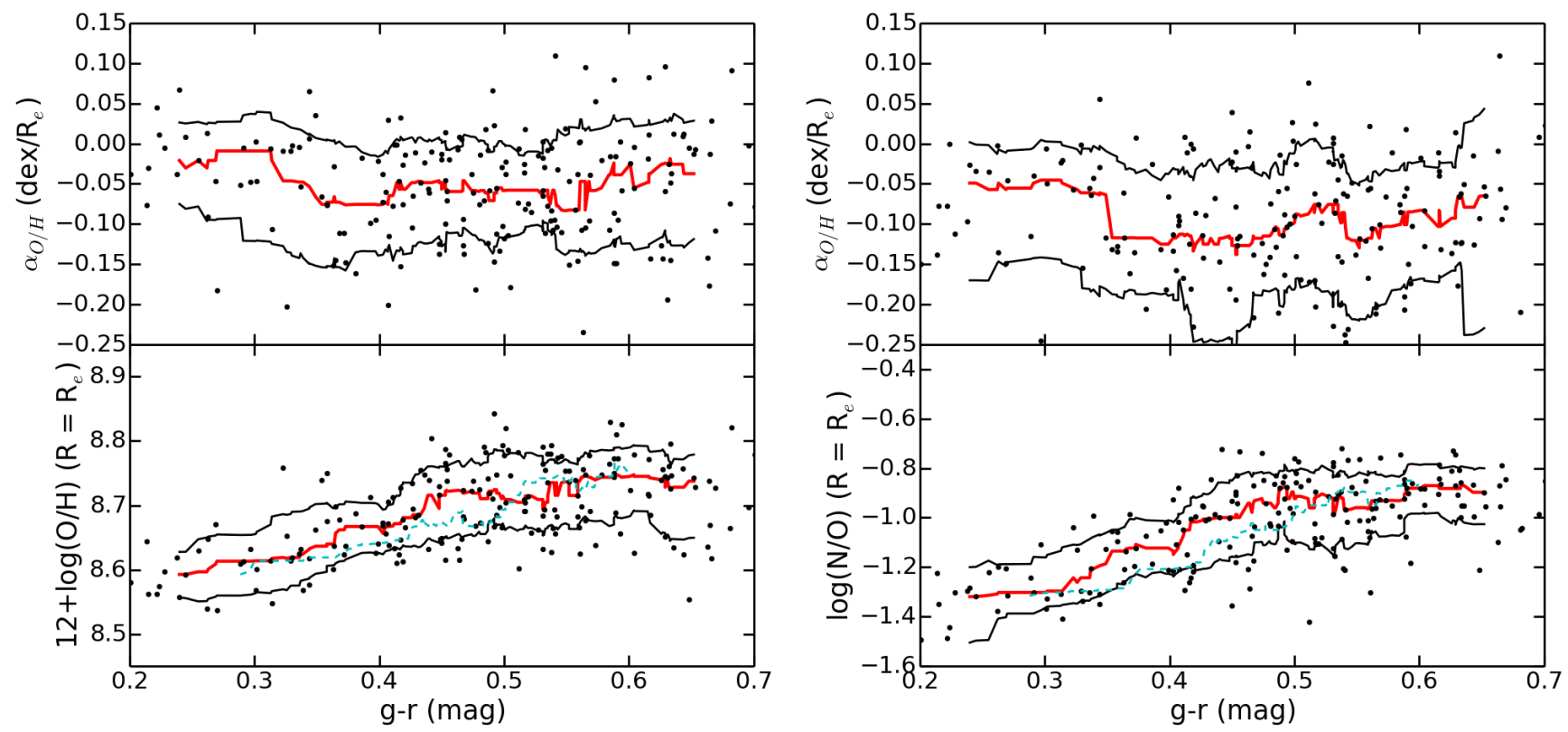

Fig. 18. Relation between the colour index $g-r$ and the derived slopes and the characteristic values at the effective radius for $\mathrm{O} / \mathrm{H}$ (left panels) and $\mathrm{N} / \mathrm{O}$ (right panels). The red and black solid lines have the same meaning as in Fig. 12. The dashed cyan lines represent the running median, assuming the observed relation between $g-r$ and stellar mass for the same galaxies.

The same behaviour between slopes or characteristic values is observed in Fig. 18, where we analyse these quantities as a function of the integrated photometric colours $g-r$ (Walcher et al. 2014), which can be interpreted as a proxy of the relation between ongoing and past star formation, as sSFR. In the respective upper panels, no evident relation is found between the $\mathrm{O} / \mathrm{H}$ and N/O slopes and the integrated colours. On the contrary, in the lower panels bluer galaxies tend to have lower abundances and redder objects tend to have higher abundances. Again, as in the case of SFR and sSFR, this result must be interpreted in light of the MZR and the relations of the colour with stellar mass for this specific non-interacting CALIFA sample with many H II regions. When we assume the observed relation between mass and $g-r$ colour index in the figures, we cannot see significant differences with the observed pattern, which demonstrates that no additional dependence exists between colour and $Z$ or $\mathrm{N} / \mathrm{O}$.

The observed empirical relation between colours and masses in this sample goes in the same direction as pointed out for the CALIFA sample by González Delgado et al. (2015), who show that on average less massive galaxies are bluer and more massive galaxies are redder. Some works have suggested that a correlation between $\mathrm{N} / \mathrm{O}$ and the integrated colour can be indicative of the star formation history owing to the delay 

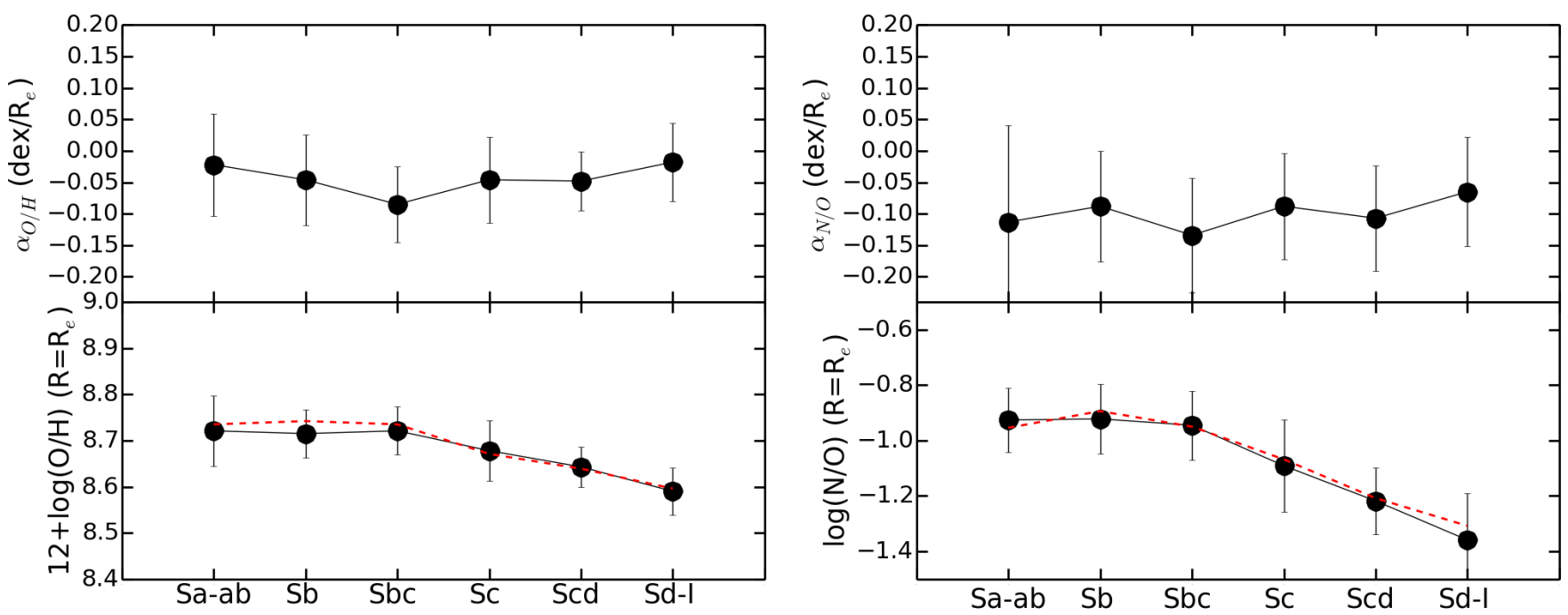

Fig. 19. Average slopes and value at the effective radius of the linear fittings in the non-interacting analysed galaxies as a function of the morphological type for $\mathrm{O} / \mathrm{H}$ (left panels) and N/O (right panels). The black solid lines connects the average points for each morphological type, while the red dashed lines correspond to the average typical abundance ratios found at the average stellar mass of each type.

Table 3. Average slopes and values at the effective radius for the linear fittings of $\mathrm{O} / \mathrm{H}$ and $\mathrm{N} / \mathrm{O}$ in the analysed CALIFA galaxies for categories attending the observed morphology and the presence of a bar.

\begin{tabular}{ccccccc}
\hline \hline Type & Number & $\%$ & $\begin{array}{c}\alpha_{\mathrm{O} / \mathrm{H}} \\
\left(\mathrm{dex} / R_{\mathrm{e}}\right)\end{array}$ & $\begin{array}{c}12+\log (\mathrm{O} / \mathrm{H}) \\
\left(\text { at } R_{\mathrm{e}}\right)\end{array}$ & $\begin{array}{c}\alpha_{\mathrm{N} / \mathrm{O}} \\
\left(\mathrm{dex} / R_{\mathrm{e}}\right)\end{array}$ & $\begin{array}{c}\log (\mathrm{N} / \mathrm{O}) \\
\left(\text { at } R_{\mathrm{e}}\right)\end{array}$ \\
\hline & & \multicolumn{5}{c}{ Morphology } \\
\hline Sa-ab & 17 & 8.5 & $-0.022 \pm 0.081$ & $8.721 \pm 0.076$ & $-0.113 \pm 0.154$ & $-0.926 \pm 0.154$ \\
$\mathrm{Sb}$ & 44 & 21.9 & $-0.046 \pm 0.072$ & $8.715 \pm 0.052$ & $-0.088 \pm 0.088$ & $-0.922 \pm 0.125$ \\
$\mathrm{Sbc}$ & 58 & 28.9 & $-0.085 \pm 0.060$ & $8.721 \pm 0.052$ & $-0.134 \pm 0.091$ & $-0.945 \pm 0.124$ \\
$\mathrm{Sc}$ & 37 & 18.4 & $-0.046 \pm 0.068$ & $8.678 \pm 0.066$ & $-0.088 \pm 0.084$ & $-1.091 \pm 0.166$ \\
$\mathrm{Scd}$ & 26 & 12.9 & $-0.048 \pm 0.047$ & $8.643 \pm 0.044$ & $-0.107 \pm 0.084$ & $-1.278 \pm 0.120$ \\
$\mathrm{Sd}-\mathrm{I}$ & 19 & 9.4 & $-0.018 \pm 0.062$ & $8.590 \pm 0.051$ & $-0.065 \pm 0.087$ & $-1.359 \pm 0.169$ \\
\hline & & & \multicolumn{5}{c}{ Presence of a bar } \\
\hline A & 89 & 44.3 & $-0.068 \pm 0.067$ & $8.680 \pm 0.068$ & $-0.102 \pm 0.091$ & $-1.071 \pm 0.213$ \\
$\mathrm{AB}$ & 53 & 26.4 & $-0.061 \pm 0.075$ & $8.678 \pm 0.067$ & $-0.109 \pm 0.099$ & $-1.061 \pm 0.176$ \\
$\mathrm{~B}$ & 59 & 29.4 & $-0.041 \pm 0.061$ & $8.710 \pm 0.073$ & $-0.100 \pm 0.101$ & $-0.979 \pm 0.182$ \\
\hline
\end{tabular}

in the ejection of $\mathrm{N}$ to the ISM (e.g. dwarf irregular galaxies, van Zee \& Haynes 2006; López-Sánchez \& Esteban 2010). However, in our sample, as most of the observed $\mathrm{N}$ has a secondary origin, the correlation with colour is totally understood in terms of the MNOR and the M-sSFR relation.

\subsection{Relation with morphological type}

In this subsection we analyse the possible link between the calculated slopes and the characteristic $\mathrm{O} / \mathrm{H}$ and $\mathrm{N} / \mathrm{O}$ values with the morphological type for the 201 galaxies of our CALIFA sample of non-interacting galaxies. The morphological classification was performed by eye, based on independent analysis by members of the CALIFA collaboration. The results are compatible with other photometric indexes and are described in detail in Walcher et al. (2014).

The results for the averages of slopes and characteristic abundance values at the effective radius for the different morphological types are listed in Table 3 and are also represented in Fig. 19. As can be seen in the respective upper panels, there is a slight trend towards shallower gradients both for $\mathrm{O} / \mathrm{H}$ and $\mathrm{N} / \mathrm{O}$ for late-type galaxies $\left(\alpha_{\mathrm{O} / \mathrm{H}}=-0.018 \mathrm{dex} / R_{\mathrm{e}}\right.$ and $\alpha_{\mathrm{N} / \mathrm{O}}=-0.065 \mathrm{dex} / R_{\mathrm{e}}$ for $\mathrm{Sd}$ galaxies). At the same time, for $\mathrm{O} / \mathrm{H}$ there is also a trend towards shallower gradients for early-type galaxies $\left(\alpha_{\mathrm{O} / \mathrm{H}}=-0.022 \mathrm{dex} / R_{\mathrm{e}}\right.$ in Sa galaxies). This result agrees with the trend found in Sánchez et al. (2014), who found a slightly shallower gradient on average for very early spiral galaxies and also with the results found by Vila-Costas \& Edmunds (1992) and Zaritsky et al. (1994) who find steeper gradients in the intermediate-type galaxies. A similar result is found for the slope of the gradient of the stellar properties by González Delgado et al. (2015) in the CALIFA sample.

Regarding the abundance values at the effective radius, considered as characteristic of the metal content of each galaxy, there is an evident decrease in the $\mathrm{O} / \mathrm{H}$ value, and this is even more evident for $\mathrm{N} / \mathrm{O}$ when we move from early- to late-type galaxies (i.e. $\mathrm{O} / \mathrm{H}$ is reduced in $0.14 \mathrm{dex}$ and $\mathrm{N} / \mathrm{O}$ in $0.35 \mathrm{dex}$ from the early to late types). This same pattern was already pointed out by Roberts \& Haynes (1994).

However, this result must be inspected in light of the relation between the typical metal content of a galaxy and its stellar mass, which is explored in subsections above. In this sample, there is a bias in the sense that late-type galaxies tend to have lower masses on average than early-type galaxies. In the lower panels 
of Fig. 19, we also show the median values for $\mathrm{O} / \mathrm{H}$ and $\mathrm{N} / \mathrm{O}$ at the average stellar mass of each morphological type group. As can be seen, no apparent difference appears between the expected values at the average stellar mass and the found value at a specific Hubble type (i.e. the mean difference is lower than 0.02 dex both for $\mathrm{O} / \mathrm{H}$ and $\mathrm{N} / \mathrm{O}$ ).

Then we can conclude that the observed relation between slopes or characteristic values with the morphological types are due to the combined effect of the MZR or MNOR and the mass-morphological type relation of this sample. This relation has been also observed for the CALIFA sample of galaxies by González Delgado et al. (2015) by means of the analysis of the spatial distribution of the stellar properties of the galaxies.

\subsection{Relation with the presence of a bar}

It has been proposed that the presence of a bar can be an element that is important to explain the flattening of gradients of chemical abundances in spiral galaxies. Vila-Costas \& Edmunds (1992) and Zaritsky et al. (1994) find this trend in their samples of spiral galaxies and they claim that this could be the consequence of the presence of gas flows driven by the bars that make the metal distribution homogenise throughout the discs.

The inspection of our sample for the presence of bars was performed by eye by members of the CALIFA collaboration, and it is described elsewhere in detail in Walcher et al. (2014). Three different groups were defined, following the classical scheme: (A) galaxies with no bar; (AB) galaxies that may have a bar, but it is not clearly visible; and (B) clearly barred galaxies. The visual classification was cross-checked with an automatic search for bars and this produced very similar results.

The results for the averages of slopes and characteristic abundance values at the effective radius for the three analysed categories are listed in Table 3. As can be seen, no significant differences exist between the average slopes either for $\mathrm{O} / \mathrm{H}$ or for $\mathrm{N} / \mathrm{O}$ as a function of the presence of a bar in this sample of galaxies, although there is a slight trend towards flatter $\mathrm{O} / \mathrm{H}$ gradients in barred galaxies. The difference in the N/O slopes of barred and unbarred galaxies is even smaller. This slight difference within the errors is still obtained when galaxies with an inclinations $>70^{\circ}$ are ruled out, as the detection of a bar is more difficult in these objects.

The possible abundance gradient flattening as a function of the presence of a bar is not observed by Sánchez et al. (2014), Marino et al. (2016), who use some of the same data but a different methodology for the calculation of $\mathrm{O} / \mathrm{H}$. A similar result is obtained by Sánchez-Blázquez et al. (2014) for a subsample of face-on spiral galaxies, where no difference is found for the gradients of stellar age and $Z$ as a function of the presence of a bar.

Regarding the characteristic chemical abundance ratios at the effective radius, there are not apparent large differences between the $\mathrm{O} / \mathrm{H}$ values found for barred and unbarred galaxies, but a slight trend towards higher $\mathrm{N} / \mathrm{O}$ values for barred galaxies is found. This difference is not statistically significant, but it cannot be because of any bias in the stellar masses of the three subsamples defined as a function of the presence of a bar. The mean stellar mass for the three subgroups are $\log \left(M / M_{\odot}\right)=10.01$ for A galaxies, 10.08 for $\mathrm{AB}$, and 10.23 for $\mathrm{B}$, which would represent an enhancement of $0.01 \mathrm{dex}$ for $\mathrm{O} / \mathrm{H}$ and $0.04 \mathrm{dex}$ for $\mathrm{N} / \mathrm{O}$, according to the results described in the above subsections. On the other hand, these results agree with the differences of chemical abundances in the centres of barred galaxies found by Florido et al. (2015), which point to an enhancement of N/O in barred galaxies, while they do not find any difference when the studied ratio is $\mathrm{O} / \mathrm{H}$. This difference in $\mathrm{N} / \mathrm{O}$ and not in $\mathrm{O} / \mathrm{H}$ in the centres of barred galaxies could be due to a different star formation efficiency in the inner parts of galaxies as a consequence of the influence of bar-driven flows of gas.

\section{An insight into flat and inverted gradients}

A non-negligible fraction of the 201 non-interacting galaxies in the CALIFA survey studied in this paper show flat or positive gradients of $\mathrm{O} / \mathrm{H}$ and/or $\mathrm{N} / \mathrm{O}$. Given the uncertainties for the emission-line fluxes that we used, as well as for those from the methodology used to derive the abundances, many of these slopes have large associated errors, but the fraction of galaxies with an inverted slope varies from $10 \%$ up to $19 \%$ in the case of $\mathrm{O} / \mathrm{H}$ and from $4.5 \%$ up to $10 \%$ in the case of $\mathrm{N} / \mathrm{O}$ depending on the errors of the slopes. We do not find a complete coincidence between the galaxies that present inverted gradients of $\mathrm{O} / \mathrm{H}$ and $\mathrm{N} / \mathrm{O}$; i.e. only 7 out of the 20 galaxies with an inverted N/O gradient also have an inverted $\mathrm{O} / \mathrm{H}$ gradient.

As shown in previous sections, there is a slight, although not statistically significant, trend towards flatter gradients in less luminous and massive galaxies and in the earliest morphological types. However, there is not any clear difference between the average integrated properties of the galaxies with an inverted gradient as compared with the whole sample. The average absolute $g$ luminosity and stellar mass for the 201 galaxies are -20.41 mag and $10^{10.09} M_{\odot}$, respectively, while these numbers are only marginally different for galaxies with an inverted $\mathrm{O} / \mathrm{H}$ $\left(-20.13 \mathrm{mag}\right.$ and $\left.10^{9.97} M_{\odot}\right)$ or N/O gradient $(-20.36 \mathrm{mag}$ and $\left.10^{10.07} M_{\odot}\right)$.

For the rest of the integrated properties, the observed relations with abundance slopes appear to be caused by the relation with the stellar mass. Therefore, in these other properties, there is not much difference between the whole sample and the subset of galaxies with an inverted $\mathrm{O} / \mathrm{H}$ or $\mathrm{N} / \mathrm{O}$ gradient. The average colours, morphological type, and classes as a function of the presence of a bar are almost identical for galaxies with inverted and negative gradients.

It has been proposed that the flows of gas throughout and across the galaxy discs can sensibly alter the observed radial distribution of the metals (e.g. Kewley et al. 2010). In the case of our sample, interacting objects were ruled out, but we should not discard the effect of minor mergers or past interactions in a recent period, after which the usual gradient shape has not yet been recovered (e.g. Miralles-Caballero et al. 2014). Positive abundance gradients have been also observed in low-mass gasrich galaxies where a radial gas redistribution has possibly taken place (e.g. NGC 2915, Werk et al. 2010).

In this sense, a flat or inverted gradient can be the evidence of an absence of correlation of the radial abundance distribution to a greater extent than the existence of a homogeneous $Z$ throughout the disc. For our sample the mean correlation coefficient of the calculated linear fittings for galaxies with a negative gradient is -0.29 for $\mathrm{O} / \mathrm{H}$ and -0.52 for $\mathrm{N} / \mathrm{O}$, while for inverted gradients the mean correlation coefficient is +0.20 for $\mathrm{O} / \mathrm{H}$ and only +0.04 for $\mathrm{N} / \mathrm{O}$. This implies that positive gradients observed for $\mathrm{N} / \mathrm{O}$ are mostly a consequence of a random abundance distribution throughout the discs. On the contrary, this is not so clearly observed in the case of $\mathrm{O} / \mathrm{H}$, once taken $\mathrm{N} / \mathrm{O}$ into account for the derivation of $\mathrm{O} / \mathrm{H}$. 
The simulations support the existence of positive gradients of $Z$ after an interaction both in the local Universe (Rupke et al. 2010) and at larger $z$ (Tissera et al. 2016) and the drop of $Z$ in the centres of galaxies after the fall of unprocessed gas (e.g. Lee et al. 2004). The fall of metal-poor gas from the exterior to explain the inverted gradients could be supported by the fact that the characteristic average $\mathrm{O} / \mathrm{H}$ at the effective radius in these galaxies is lower. In Table 2 we see that in these galaxies $\mathrm{O} / \mathrm{H}$ is 0.04 dex lower on average. Besides, the $\mathrm{N} / \mathrm{O}$ characteristic value is almost identical to inverted $\mathrm{O} / \mathrm{H}$ gradient, which would partially support the argument of the infall of metal-poor gas, as N/O tio is not expected to change substantially when a gas interchange is produced with the IGM medium (Edmunds 1990). On the contrary, galaxies with inverted N/O gradient present sensibly lower N/O characteristic values, which could be indicative of other causes for the inversion of the gradient, which is more related to the variation of the star formation efficient.

However, the influence of these gas flows should also be observed in the derived SFR and neither for the integrated SFR nor for sSFR is any clear difference seen between galaxies with an inverted gradient and the whole sample, as shown in Sect. 6.2. At same time, we do not observed that the mean $\mathrm{H} \alpha$ luminosity of the $\mathrm{H}$ II regions in galaxies with an inverted gradient is higher $\left(L(\mathrm{H} \alpha)=10^{39.03} \mathrm{erg} / \mathrm{s}\right.$ for regions in galaxies with positive $\mathrm{O} / \mathrm{H}$ slope and $10^{39.13} \mathrm{erg} / \mathrm{s}$ for all $\mathrm{H}$ II regions) as seen in Fig. 16. The mean $\mathrm{O} / \mathrm{H}$ and $\mathrm{N} / \mathrm{O}$ are also the same in both sets of $\mathrm{H}$ II regions.

An inner gas transportation and redistribution from the galaxies centres towards the outer positions could be also supported by the observation of the flattening of the $Z$ gradient beyond $2 \cdot R_{\mathrm{e}}$ (e.g. Bresolin et al. 2009; Rosales-Ortega et al. 2011; Marino et al. 2012; Sánchez et al. 2014). Additionally, it has been observed that a decrease of $Z$ in the inner regions of spiral galaxies in the radial range $0.3-0.5 R_{\mathrm{e}}$ (e.g. Rosales-Ortega et al. 2011; Sánchez et al. 2012b, 2014) is possibly related to an accumulation of gas in rings, which can make he resulting slopes of the $Z$ gradients flatter. For this work, no restriction was made in the fitted radial range Nevertheless, these two factors can have a certain influence on the fraction of observed flat or inverted gradients in our sample. By restricting the analysed $\mathrm{H}$ II regions to the range $0.5-2.1 R_{\mathrm{e}}$, the number of galaxies with at least $10 \mathrm{H}$ II regions is reduced to 147 . For these the average slopes are $-0.065 \mathrm{dex} / R_{\mathrm{e}}$ for $\mathrm{O} / \mathrm{H}$ and $-0.115 \mathrm{dex} / R_{\mathrm{e}}$ for $\mathrm{N} / \mathrm{O}$, which are slightly more pronounced than for the whole galaxy. The respective fractions of objects with an inverted gradient oscillates depending on the error from $9 \%$ up to $17 \%$ in the case of $\mathrm{O} / \mathrm{H}$ and from $2 \%$ up to $12 \%$ for N/O.

Another factor that can have an influence on the calculations and thus can bias the results towards flatter abundance gradients is the inclination of the galaxies. Sánchez et al. (2014) only use objects with an inclination that is lower than $70^{\circ}$ to sample $\mathrm{H}$ II regions at all galactocentric distances and to avoid a bias towards the regions at outer distances. We calculated the inclinations using the ratio of the semi-axis $(b / a)$ measured over the photometric growth curves given by Walcher et al. (2014) and we used the same restriction as Sánchez et al. (2014), which reduces the sample of analysed galaxies to 113 . The average slopes for these are $-0.063 \mathrm{dex} / R_{\mathrm{e}}$ for $\mathrm{O} / \mathrm{H}$ and $-0.125 \mathrm{dex} / R_{\mathrm{e}}$ for $\mathrm{N} / \mathrm{O}$. The respective fractions of objects with an inverted gradient in this subsample ranges from $6 \%$ up to $15 \%$ for $\mathrm{O} / \mathrm{H}$ and from $1 \%$ up to $4 \%$ for $\mathrm{N} / \mathrm{O}$. By combining this subsample of objects with an inclination that is lower than $70^{\circ}$ with the fitting in the radial range $0.5-2.1 R_{\mathrm{e}}$ (98 galaxies), the average slope is of $-0.062 \mathrm{dex} / R_{\mathrm{e}}$ for $\mathrm{O} / \mathrm{H}$ and $-0.113 \mathrm{dex} / R_{\mathrm{e}}$ for $\mathrm{N} / \mathrm{O}$. The fraction of galaxies with an inverted gradients ranges from $11 \%$ up to $19 \%$ for $\mathrm{O} / \mathrm{H}$ and from $1 \%$ up to $8 \%$ for $\mathrm{N} / \mathrm{O}$. For this same subsample, by making calculations assuming the $\mathrm{O} / \mathrm{H}$ values derived by $\mathrm{HCM}$ without a previous determination of $\mathrm{N} / \mathrm{O}$ and assuming a typical $\mathrm{O} / \mathrm{H}-\mathrm{N} / \mathrm{O}$ relation, we obtained an average $\mathrm{O} / \mathrm{H}$ slope of $-0.095 \mathrm{dex} / R_{\mathrm{e}}$ and a very high $p$ value in the Lilliefors normality test $(0.94)$; this approximates the results obtained by Sánchez et al. (2014) very accurately.

\section{Summary and conclusions}

In this work we studied the $\mathrm{O} / \mathrm{H}$ and $\mathrm{N} / \mathrm{O}$ abundance ratios of the ionised gas phase in individual H II regions and the radial distributions of these abundances across the discs in a sample of 351 galaxies using the spatially resolved IFS data from the CALIFA survey Sánchez et al. (2012a).

The abundances were derived in the selected star-forming $\mathrm{H}$ II regions using the semi-empirical code HCM (Pérez-Montero 2014), based on photoionisation models. This code permits the derivation of $\mathrm{O} / \mathrm{H}, \mathrm{N} / \mathrm{O}$ and $\log U$, covering a wide range of input values and is totally consistent with the direct method when the resulting abundances are compared in samples of objects with measured electron temperature, as in Pérez-Montero (2014). This method takes the dependence of [N II] emission lines on nitrogen abundances into account before its use to derive $Z$, so it is adequate to independently study $\mathrm{O} / \mathrm{H}$ and $\mathrm{N} / \mathrm{O}$ without considering any previous assumption of their relation.

The analysis of the $\mathrm{O} / \mathrm{H}-\mathrm{N} / \mathrm{O}$ relation for the selected starforming regions reveals a large dispersion with a higher slope in relation to the sample of objects used to calibrate the $\mathrm{O} / \mathrm{H}-\mathrm{N} / \mathrm{O}$ in the models when N/O cannot be calculated, but this analysis is in agreement with a general scenario of higher $\mathrm{N} / \mathrm{O}$ for higher $\mathrm{O} / \mathrm{H}$ in the regime of production of secondary N. Nevertheless, it cannot be ruled out that the discrepancies found between the CALIFA H II regions and the $\mathrm{H}$ II regions with the direct method can be due to emission-line measurements or uncertainties in the methodology used. The range of variation of N/O in this sample $[-1.8,-0,5]$ is much larger than for $\mathrm{O} / \mathrm{H}[8.4,8.9]$ with a similar associated error, which supports the idea that N/O can be a much more accurate indicator of the chemical status of an object than $\mathrm{O} / \mathrm{H}$.

The analysis of the radial distributions both for $\mathrm{O} / \mathrm{H}$ and $\mathrm{N} / \mathrm{O}$ normalised to the effective radius in those non-interacting galaxies with at least 10 star-forming H II regions (201 galaxies) shows that most of the galaxies present a negative gradient, although these galaxies have a large dispersion with a mean slope $\alpha=-0.053 \pm 0.068 \mathrm{dex} / R_{\mathrm{e}}$ for $\mathrm{O} / \mathrm{H}$ and $\alpha=$ $-0.104 \pm 0.096 \mathrm{dex} / R_{\mathrm{e}}$ for N/O. In fact, a non-negligible fraction of galaxies present flat or even inverted gradients (at least a $10 \%$ for $\mathrm{O} / \mathrm{H}$ and $4 \%$ for $\mathrm{N} / \mathrm{O}$ ). A correlation between $\mathrm{O} / \mathrm{H}$ and $\mathrm{N} / \mathrm{O}$ slopes exists $\left(\rho_{\mathrm{s}}=0.39\right)$ but with large dispersion and even different combinations of $\mathrm{O} / \mathrm{H}$ and $\mathrm{N} / \mathrm{O}$ gradients can co-exist. This fact reveals the importance of a previous determination of $\mathrm{N} / \mathrm{O}$ when $[\mathrm{N} \mathrm{II}]$ lines are used to derive $\mathrm{O} / \mathrm{H}$.

Contrary to individual $\mathrm{H}$ II regions for which the relation between $\mathrm{O} / \mathrm{H}$ and $\mathrm{N} / \mathrm{O}$ shows a very large dispersion and a relatively low correlation $\left(\rho_{\mathrm{s}}=0.37\right)$, the characteristic $\mathrm{O} / \mathrm{H}$ and $\mathrm{N} / \mathrm{O}$ values (i.e. the values of the linear fittings at the effective radius $)$ are well correlated $\left(\rho_{\mathrm{s}}=0.80\right)$ and have a very low dispersion (i.e. the standard deviation of the residuals is of 0.12 dex); this supports the idea that the global chemical conditions of a galaxy is mostly related with their integrated properties regardless of its inner spatial variations. This has been also observed for 
the CALIFA sample of galaxies for the stellar population properties (González Delgado et al. 2015).

When analysing the distribution of slopes as a function of the galactic integrated properties, we see that galaxies with low stellar masses and absolute $g$ luminosities tend to have flatter gradients, once normalised by the effective radius for $\mathrm{O} / \mathrm{H}$ and $\mathrm{N} / \mathrm{O}$, but this result is not statistically significant. The same occurs with the morphological type, as early and late spiral galaxies have flatter gradients than intermediate-type objects. In addition, no significant variation of the median slopes is found as a function of the presence of a bar. These results agree with the analysis made for other samples of these CALIFA galaxies using other strong-line methods for H II regions (Sánchez et al. 2014; Marino et al. 2016), in the analysis spaxel-to-spaxel (Sánchez-Menguiano et al. 2016) or even from the analysis of the stellar abundances (Sánchez-Blázquez et al. 2014).

Regarding the characteristic $\mathrm{O} / \mathrm{H}$ and N/O values at the effective radius, the MZR and MNOR are recovered with very low dispersion (i.e. the standard deviation of the residuals to a quadrant fitting is around 0.03 dex for both abundance ratios), which confirms that these global relations are better obtained when the characteristic value of a galaxy, independent of the inner spatial variations, is used. We also observe clear relations between these characteristic abundances for each galaxy and other integrated properties, but all of these can be interpreted in light of the MZR or the MNOR. In this way, although clear relations are observed between both $\mathrm{O} / \mathrm{H}$ or $\mathrm{N} / \mathrm{O}$ with the integrated present-day SFR and SSFR, these relations result from the M-SFR relation followed by all these objects. The observed M-SFR-Z-N/O relation makes it possible to observe a certain correlation between $\mathrm{L}(\mathrm{H} \alpha)$ and $\mathrm{O} / \mathrm{H}$ (or $\mathrm{N} / \mathrm{O}$ ) for the individual $\mathrm{H}$ II regions of the galaxies in this sample. This absence of an additional dependence between $\mathrm{O} / \mathrm{H}$ and SFR, as well as with $\mathrm{N} / \mathrm{O}$ in the regime of secondary production of nitrogen, agrees with the results found by Sánchez et al. (2013) for the sample of H II regions in CALIFA galaxies. We also observe clear correlations between abundances with integrated colours and morphology but, again, these are understood in terms of a sequence of earlier types and redder colours for more massive galaxies in this sample as also seen by (González Delgado et al. 2015).

Finally, we explored the nature of those objects with flat and inverted gradients of $Z$ and $\mathrm{N} / \mathrm{O}$, but we did not find any special feature in these objects that can explain the inversion of the chemical gradient in the whole radial range. No significant variations in the average slopes or the fraction of inverted gradients are found when additional restrictions are taken that relate to the fitted radial range or the inclination of the galaxies, although these further reduce the proportion of these gradients. A deeper inspection of these galaxies considering the past history of minor interactions, gas accretion, and radial flows should be considered to understand that causes of these flat radial abundance distributions.

Acknowledgements. This study makes uses of the data provided by the Calar Alto Legacy Integral Field Area (CALIFA) survey (http://califa.caha. es/). CALIFA is the first legacy survey being performed at Calar Alto. The CALIFA collaboration would like to thank the IAA-CSIC and MPIA-MPG as major partners at the observatory, and CAHA itself, for the unique access to telescope time and support in manpower and infrastructures. The CALIFA collaboration also thanks the CAHA staff for their dedication to this project. E.P.M., J.M.V., C.K., S.P., and J.I.P. acknowledge support from the Spanish MICINN through grants AYA2010-21887-C04-01 and AYA2013-47742-C4-1-P and the Junta de Andalucía for grant EXC/2011 FQM-7058. R.G.B., R.G.D., and E.P. acknowledge support from grants AYA2014-57490-P and JA-FQM-2828.
Support for L.G. is provided by the Ministry of Economy, Development, and Tourism's Millennium Science Initiative through grant IC120009, awarded to The Millennium Institute of Astrophysics, MAS. L.G. acknowledges support by CONICYT through FONDECYT grant 3140566. This research made use of python (http: //www . python.org), of Matplotlib (Hunter 2007), a suite of open-source python modules that provides a framework for creating scientific plots. We also thank Dr. Christophe Morisset for many discussions and constructive comments that have helped to improve the original manuscript.

\section{References}

Alloin, D., Collin-Souffrin, S., Joly, M., \& Vigroux, L. 1979, A\&A, 78, 200 Amorín, R. O., Pérez-Montero, E., \& Vílchez, J. M. 2010, ApJ, 715, L128 Baldwin, J. A., Phillips, M. M., \& Terlevich, R. 1981, PASP, 93, 5

Barrera-Ballesteros, J. K., Sánchez, S. F., García-Lorenzo, B., et al. 2015, A\&A, 579, A45

Bresolin, F., Ryan-Weber, E., Kennicutt, R. C., \& Goddard, Q. 2009, ApJ, 695, 580

Bresolin, F., Kennicutt, R. C., \& Ryan-Weber, E. 2012, ApJ, 750, 122

Brinchmann, J., Charlot, S., White, S. D. M., et al. 2004, MNRAS, 351, 1151

Bryant, J. J., Owers, M. S., Robotham, A. S. G., et al. 2015, MNRAS, 447, 2857

Bundy, K., Bershady, M. A., Law, D. R., et al. 2015, ApJ, 798, 7

Cardelli, J. A., Clayton, G. C., \& Mathis, J. S. 1989, ApJ, 345, 245

Carton, D., Brinchmann, J., Wang, J., et al. 2015, MNRAS, 451, 210

Cid Fernandes, R., Stasińska, G., Mateus, A., \& Vale Asari, N. 2011, MNRAS 413, 1687

Cid Fernandes, R., Pérez, E., García Benito, R., et al. 2013, A\&A, 557, A86

Contini, T., Garilli, B., Le Fèvre, O., et al. 2012, A\&A, 539, A91

Cresci, G., Mannucci, F., Maiolino, R., et al. 2010, Nature, 467, 811

Croxall, K. V., Pogge, R. W., Berg, D. A., Skillman, E. D., \& Moustakas, J. 2015, ApJ, 808, 42

Croxall, K., Pogge, R. W., Berg, D. A., Skillman, E. D., \& Moustakas, J. 2016, ApJ, 830, 4

Diaz, A. I. 1989, in Evolutionary Phenomena in Galaxies, eds. J. E. Beckman, \& B. E. J. Pagel (Cambridge University Press), 377

Dopita, M. A., \& Evans, I. N. 1986, ApJ, 307, 431

Edmunds, M. G. 1990, MNRAS, 246, 678

Ellison, S. L., Patton, D. R., Simard, L., \& McConnachie, A. W. 2008, ApJ, 672, L107

Evans, I. N., \& Dopita, M. A. 1985, ApJS, 58, 125

Falcón-Barroso, J., Sánchez-Blázquez, P., Vazdekis, A., et al. 2011, A\&A, 532, A95

Ferland, G. J., Porter, R. L., van Hoof, P. A. M., et al. 2013, Rev. Mex. Astron. Astrofis., 49, 137

Florido, E., Zurita, A., Pérez, I., et al. 2015, A\&A, 584, A88

García-Benito, R., Zibetti, S., Sánchez, S. F., et al. 2015, A\&A, 576, A135

Garnett, D. R., \& Shields, G. A. 1987, ApJ, 317, 82

Garnett, D. R., Shields, G. A., Skillman, E. D., Sagan, S. P., \& Dufour, R. J. 1997, ApJ, 489, 63

González Delgado, R. M., García-Benito, R., Pérez, E., et al. 2015, A\&A, 581, A103

Ho, I.-T., Kudritzki, R.-P., Kewley, L. J., et al. 2015, MNRAS, 448, 2030

Hunter, J. D. 2007, Comput. Sci. Eng., 9, 90

Kauffmann, G., Heckman, T. M., Tremonti, C., et al. 2003, MNRAS, 346, 1055

Kehrig, C., Monreal-Ibero, A., Papaderos, P., et al. 2012, A\&A, 540, A11

Kelz, A., Verheijen, M. A. W., Roth, M. M., et al. 2006, PASP, 118, 129

Kewley, L. J., Dopita, M. A., Sutherland, R. S., Heisler, C. A., \& Trevena, J. 2001, ApJ, 556, 121

Kewley, L. J., Groves, B., Kauffmann, G., \& Heckman, T. 2006, MNRAS, 372, 961

Kewley, L. J., Rupke, D., Zahid, H. J., Geller, M. J., \& Barton, E. J. 2010, ApJ, 721, L48

Köppen, J., \& Hensler, G. 2005, A\&A, 434, 531

Lamareille, F., Mouhcine, M., Contini, T., Lewis, I., \& Maddox, S. 2004, MNRAS, 350, 396

Lara-López, M. A., Cepa, J., Bongiovanni, A., et al. 2010, A\&A, 521, L53

Lee, J. C., Salzer, J. J., \& Melbourne, J. 2004, ApJ, 616, 752

Lequeux, J., Peimbert, M., Rayo, J. F., Serrano, A., \& Torres-Peimbert, S. 1979, A\&A, 80, 155

Lilly, S. J., Le Fèvre, O., Renzini, A., et al. 2007, ApJS, 172, 70

López-Sánchez, Á. R., \& Esteban, C. 2010, A\&A, 517, A85

López-Sánchez, Á. R., Westmeier, T., Esteban, C., \& Koribalski, B. S. 2015, MNRAS, 450, 3381 
Mannucci, F., Cresci, G., Maiolino, R., Marconi, A., \& Gnerucci, A. 2010, MNRAS, 408, 2115

Marino, R. A., Gil de Paz, A., Castillo-Morales, A., et al. 2012, ApJ, 754, 61

Marino, R. A., Rosales-Ortega, F. F., Sánchez, S. F., et al. 2013, A\&A, 559, A114

Marino, R. A., Gil de Paz, A., Sánchez, S. F., et al. 2016, A\&A, 585, A47

Mattsson, L. 2009, in IAU Symp. 254, eds. J. Andersen, Nordströara, B. M., \& J. Bland-Hawthorn, 43

Miralles-Caballero, D., Díaz, A. I., Rosales-Ortega, F. F., Pérez-Montero, E., \& Sánchez, S. F. 2014, MNRAS, 440, 2265

Mollá, M., Vílchez, J. M., Gavilán, M., \& Díaz, A. I. 2006, MNRAS, 372, 1069

Mollá, M., García-Vargas, M. L., \& Bressan, A. 2009, MNRAS, 398, 451

Moran, S. M., Heckman, T. M., Kauffmann, G., et al. 2012, ApJ, 745, 66

Moustakas, J., \& Kennicutt, Jr., R. C. 2006, ApJS, 164, 81

Pérez-Montero, E. 2014, MNRAS, 441, 2663

Pérez-Montero, E., \& Contini, T. 2009, MNRAS, 398, 949

Pérez-Montero, E., \& Díaz, A. I. 2005, MNRAS, 361, 1063

Pérez-Montero, E., \& Vílchez, J. M. 2009, MNRAS, 400, 172

Pérez-Montero, E., Vílchez, J. M., Cedrés, B., et al. 2011, A\&A, 532, A141

Pérez-Montero, E., Contini, T., Lamareille, F., et al. 2013, A\&A, 549, A25

Pichon, C., Pogosyan, D., Kimm, T., et al. 2011, MNRAS, 418, 2493

Pilyugin, L. S., \& Grebel, E. K. 2016, MNRAS, 457, 3678

Pilyugin, L. S., Vílchez, J. M., \& Contini, T. 2004, A\&A, 425, 849

Pilyugin, L. S., Thuan, T. X., \& Vílchez, J. M. 2007, MNRAS, 376, 353

Queyrel, J., Contini, T., Kissler-Patig, M., et al. 2012, A\&A, 539, A93

Rich, J. A., Torrey, P., Kewley, L. J., Dopita, M. A., \& Rupke, D. S. N. 2012, ApJ, 753, 5

Roberts, M. S., \& Haynes, M. P. 1994, ARA\&A, 32, 115

Rosales-Ortega, F. F., Díaz, A. I., Kennicutt, R. C., \& Sánchez, S. F. 2011, MNRAS, 415, 2439

Rosales-Ortega, F. F., Sánchez, S. F., Iglesias-Páramo, J., et al. 2012, ApJ, 756 L31
Roth, M. M., Kelz, A., Fechner, T., et al. 2005, PASP, 117, 620

Rupke, D. S. N., Kewley, L. J., \& Chien, L.-H. 2010, ApJ, 723, 1255

Sánchez, S. F., García-Lorenzo, B., Jahnke, K., et al. 2006, New Astron. Rev., 49, 501

Sánchez, S. F., Rosales-Ortega, F. F., Kennicutt, R. C., et al. 2011, MNRAS, 410, 313

Sánchez, S. F., Kennicutt, R. C., Gil de Paz, A., et al. 2012a, A\&A, 538, A8

Sánchez, S. F., Rosales-Ortega, F. F., Marino, R. A., et al. 2012b, A\&A, 546, A2

Sánchez, S. F., Rosales-Ortega, F. F., Jungwiert, B., et al. 2013, A\&A, 554, A58

Sánchez, S. F., Rosales-Ortega, F. F., Iglesias-Páramo, J., et al. 2014, A\&A, 563, A49

Sánchez-Blázquez, P., Rosales-Ortega, F. F., Méndez-Abreu, J., et al. 2014, A\&A, 570, A6

Sánchez-Menguiano, L., Sánchez, S. F., Pérez, I., et al. 2016, A\&A, 587, A70

Skillman, E. D. 1989, ApJ, 347, 883

Storey, P. J., \& Hummer, D. G. 1995, MNRAS, 272, 41

Thuan, T. X., Pilyugin, L. S., \& Zinchenko, I. A. 2010, ApJ, 712, 1029

Tissera, P. B., Pedrosa, S. E., Sillero, E., \& Vilchez, J. M. 2016, MNRAS, 456, 2982

Tremonti, C. A., Heckman, T. M., Kauffmann, G., et al. 2004, ApJ, 613, 898

Troncoso, P., Maiolino, R., Sommariva, V., et al. 2014, A\&A, 563, A58

van Zee, L., \& Haynes, M. P. 2006, ApJ, 636, 214

Vazdekis, A., Sánchez-Blázquez, P., Falcón-Barroso, J., et al. 2010, MNRAS, 404, 1639

Veilleux, S., \& Osterbrock, D. E. 1987, ApJS, 63, 295

Vila-Costas, M. B., \& Edmunds, M. G. 1992, MNRAS, 259, 121

Walcher, C. J., Wisotzki, L., Bekeraité, S., et al. 2014, A\&A, 569, A1

Werk, J. K., Putman, M. E., Meurer, G. R., \& Santiago-Figueroa, N. 2011, ApJ, 735,71

Werk, J. K., Putman, M. E., Meurer, G. R., et al. 2010, ApJ, 715, 656

Zaritsky, D., Kennicutt, Jr., R. C., \& Huchra, J. P. 1994, ApJ, 420, 87 